\title{
Water Ice and Dust in the Innermost Coma of Comet 103P/Hartley 2
}

\author{
Silvia Protopapa ${ }^{a}$, Jessica M. Sunshine ${ }^{a}$, Lori M. Feaga ${ }^{a}$, Michael S. P. Kelley ${ }^{a}$, \\ Michael F. A' Hearn ${ }^{\mathrm{a}}$, Tony L. Farnham ${ }^{\mathrm{a}}$, Olivier Groussin ${ }^{\mathrm{b}}$, Sebastien Besse ${ }^{\mathrm{a}, \mathrm{c}}$, \\ Frédéric Merlin ${ }^{\text {d }}$, Jian-Yang Li ${ }^{\mathrm{e}}$ \\ ${ }^{a}$ Department of Astronomy, University of Maryland, College Park, MD, 20742, USA \\ ${ }^{b}$ Aix-Marseille Université, CNRS, LAM (Laboratoire dAstrophysique de Marseille) UMR 7326, \\ 13388, Marseille, France \\ ${ }^{c}$ ESA/ESTEC, Keplerlaan 1, Noordwijk, The Netherlands \\ ${ }^{d}$ Université Denis Diderot Paris 7, LESIA, Observatoire de Paris, France \\ e Planetary Science Institute, Tucson, AZ 85719, USA
}

\begin{abstract}
On November 4th, 2010, the Deep Impact eXtended Investigation (DIXI) successfully encountered comet 103P/Hartley 2, when it was at a heliocentric distance of 1.06 AU. Spatially resolved near-IR spectra of comet Hartley 2 were acquired in the $1.05-4.83 \mu \mathrm{m}$ wavelength range using the HRI-IR spectrometer. We present spectral maps of the inner $\sim 10$ kilometers of the coma collected 7 minutes and 23 minutes after closest approach. The extracted reflectance spectra include welldefined absorption bands near 1.5, 2.0, and $3.0 \mu \mathrm{m}$ consistent in position, bandwidth, and shape with the presence of water ice grains. Using Hapke's radiative transfer model, we characterize the type of mixing (areal vs. intimate), relative abundance, grain size, and spatial distribution of water ice and refractories. Our modeling suggests that the dust, which dominates the innermost coma of Hartley 2 and is at a temperature of $300 \mathrm{~K}$, is thermally and physically decoupled from the fine-grained water ice particles, which are on the order of $1 \mu \mathrm{m}$ in size. The strong correlation between the water ice, dust, and $\mathrm{CO}_{2}$ spatial distribution supports the
\end{abstract}


concept that $\mathrm{CO}_{2}$ gas drags the water ice and dust grains from the nucleus. Once in the coma, the water ice begins subliming while the dust is in a constant outflow. The derived water ice scale-length is compatible with the lifetimes expected for $1-\mu \mathrm{m}$ pure water ice grains at $1 \mathrm{AU}$, if velocities are near $0.5 \mathrm{~m} / \mathrm{s}$. Such velocities, about three order of magnitudes lower than the expansion velocities expected for isolated 1- $\mu \mathrm{m}$ water ice particles [Hanner, 1981; Whipple, 1951], suggest that the observed water ice grains are likely aggregates.

Keywords:

Comets, Comets, coma, Comets, composition, Comets, dust, Ices

\section{Introduction}

Comets formed beyond the $\mathrm{H}_{2} \mathrm{O}$ frost line, where ices can condense [e.g., A'Hearn et al. 2012]. A variety of processes have affected comets during their long storage in the Oort cloud and scattered disk (e.g., irradiation by energetic particles, heating by passing stars, collisions). Similarly, repeated solar heating leads to evolution of short period comets during their many passages close to the Sun. However, because most of these processes affect only the outer layer of comets, the pristine nature of the bulk of the nucleus is preserved [Bockelée-Morvan et al. 2004; Mumma et al., 1993; Stern, 2003; Weissman and Stern, 1997]. As such, comets are excellent laboratories to extend our understanding of the origin and evolution of the Solar System.

Given that comets contain the least processed primordial materials that formed the cores of the giant planets [A'Hearn, 2011], the analysis of the composition and physical state of cometary materials is critical to improve our understanding of the accretion processes that led to the formation of comet nuclei and ultimately the 
planets. Water is a key component of comets [Bockelée-Morvan et al., 2004; Bockelée-Morvan and Rickman, 1997; Feaga et al., 2007; Mumma and Charnley, 2011] and water ice has been observed from the ground and with in situ observations in the comae [Davies et al., 1997; Kawakita et al., 2004; Sunshine et al., 2011a; Yang et al., 2009], on the surfaces [Sunshine et al., 2006], and in the nearsurface interiors [Sunshine et al., 2007] of comets. An example of addressing comet nuclei formation by means of water ice characteristics is given by Sunshine et al. [2007]: the presence of very fine $(\sim 1 \mu \mathrm{m})$ water ice particles in the impact ejecta of comet Tempel 1, free of refractory impurities, led to question the interstellar dust grain model proposed by Greenberg [1998] and Greenberg and Li [1999]. The basic idea of this model is that comets formed directly through coagulation of interstellar dust. As such, the morphological structure of comet nuclei is an aggregate of presolar interstellar dust grains, which consist of a core of silicates mantled first by a shell of organic refractory material and then by a mixture of water dominated ices, which are embedded with thousands of very small (1-10 nm) carbonaceous/large molecule particles. According to Sunshine et al. [2007], it is unlikely that water ice would segregate from the superfine particles in the impact ejecta. The model by Greenberg is only one of the several models proposed, describing the underlying structure of cometary nuclei (e.g., the "fluffy aggregate" of Donn et al. [1985] and Donn and Hughes [1986], the "rubble pile model", either collisionally modified [Weissman et al., 2004] or primordial [Weissman, 1986], the "icy-glue" model of Gombosi and Houpis [1986], the "talps" or "layered pile" model by Belton et al. [2007]). All of these models consider different origins and/or evolutionary processes. Investigations of cometary nuclei at close range, which can only be achieved by space missions, are required to validate, disprove, 
or improve these various formation models.

On November 4th, 2010, the Deep Impact Flyby (DIF) spacecraft [A'Hearn et al. 2005; Hampton et al., 2005] successfully encountered comet 103P/Hartley 2 as part of its extended mission, the Deep Impact eXtended Investigation (DIXI) [A'Hearn et al., 2011]. The DIF spacecraft employs two multi-spectral imagers (MRI-VIS, HRI-VIS) and a $1.05-4.83 \mu \mathrm{m}$ near-infrared spectrometer (HRI-IR). Near-IR spectra of comet Hartley 2 were acquired for several weeks before and after closest approach (CA, at a range of $694 \mathrm{~km}$, occurred on November 4th, 2010, at 13:59:47.31 UTC with a maximum HRI-IR spatial resolution of $7 \mathrm{~m} / \mathrm{pixel}$ ). DIXI observations revealed a bi-lobed, very small (maximum length of $2.33 \mathrm{~km}$, [A'Hearn et al., 2011]), and highly active nucleus, with water ice distributed heterogeneously, in specific areas on the surface [Sunshine et al. 2011b] and in the coma [Protopapa et al. 2011; Sunshine et al., 2011a].

In this paper, we present a detailed analysis of the composition and texture of water ice and refractories in the inner-most coma of Hartley 2, within a few kilometers of the surface, using DI HRI-IR data. We investigate the physical makeup of the water ice grains, with the goal of providing more observational constraints to help us have a better understanding of the accretion process that led to the formation of comet nuclei. We investigate the role of the gas in delivering the water ice and dust to the coma of Hartley 2 and how the emitted material evolves after it leaves the nucleus.

\section{Observations}

Spatially resolved near-IR spectra of comet Hartley 2 were acquired in the $1.05-4.83 \mu \mathrm{m}$ wavelength range using the HRI-IR spectrometer [Hampton et al. 
2005]. HRI-IR data were collected from 01 October 2010 to 26 November 2010. In this paper, only a subset of data is analyzed. In particular, we present HRIIR spatially resolved scans of comet Hartley 2 collected 7 (ID 5006000) and 23 (ID 5007002) minutes post-CA. Table 1 lists the main characteristics of these two scans.

The IR data are calibrated using the DI science data pipeline [Klaasen et al., 2008, 2013] and are available in the NASA Planetary Data System (PDS) archive [McLaughlin et al., 2013]. The standard steps of the pipeline processing include linearization, dark subtraction, flat fielding, conversion from DN to radiance units ( $\mathrm{W} \mathrm{m}{ }^{-2} \mathrm{sr}^{-1} \mu \mathrm{m}^{-1}$ ), and bad pixel masking. These data have been recalibrated since the work by A'Hearn et al. [2011], although the differences are not dramatic. The 5006000 and 5007002 radiance maps are shown in panels (a) and (b) of Figure 1. respectively, compared with the HRIVIS and MRI-VIS context images (with characteristics given in Table 2). Because the HRI-IR instrument is a scanning slit spectrometer, reconstructed spatial information is susceptible to errors from jumps in spacecraft attitude due to impacting particles or autonomous operations. A mismatch in the nucleus shape between the IR scan acquired 7 min post-CA and the corresponding visible context images was observed, possibly caused by very small grains hitting the spacecraft at high speed. We have corrected this mismatch by shifting the bottom 14 rows of the 56-row IR scan by 1 pixel to the left and the top 21 rows by 1 pixel toward the right and bottom (with respect to the orientation of Figure 11.

Both the 5006000 and 5007002 scans contain the nucleus of Hartley 2 and the surrounding coma. The nucleus is in roughly the same orientation in the two scans. The precession of the long axis of the nucleus around the angular momen- 
tum vector has a period of 18.4 hours at encounter [A'Hearn et al., 2011; Belton et al. 2013]; therefore in 16 min separating the two scans, the rotation of the nucleus is negligible with regards to coma studies, as is the change of the spacecraft line of sight. In both scans, there are jets off the end of the smaller lobe of the nucleus and beyond the terminator along the lower edge of the larger lobe (Figure 1).

The nucleus of Hartley 2 needs to be masked in order to focus our analysis on the water ice and refractories in the inner-most coma. The dashed red line in Figure 1 outlines the illuminated portion of the cometary nucleus, including the scattered light near the limb, that is excluded from our analysis. This mask was defined at long wavelengths $(\lambda \geq 3.5 \mu \mathrm{m})$, where the thermal emission of the nucleus dominates over the signal from the dust in the coma, allowing the nucleus and coma to be disentangled. The nucleus contour is defined by radiance values in the 450 channel $(\sim 4.1 \mu \mathrm{m})$ greater than $0.16 \mathrm{~W} \mathrm{~m}^{-2} \mathrm{sr}^{-1} \mu \mathrm{m}^{-1}$ in order to match the nucleus as seen in the HRI-VIS and MRI-VIS images acquired at the same time as the IR data.

\section{Spectral Modeling}

\subsection{Reflectance}

Once the flux is calibrated into radiance, $I$, from the pipeline, we can convert it into reflectance, $R$, by

$$
R(\lambda)=\frac{\pi I(\lambda) r^{2}}{F_{\odot}(\lambda)}
$$

where $F_{\odot}(\lambda)$ is the solar flux at $1 \mathrm{AU}$, and $r$ is the heliocentric distance, in our case $1.06 \mathrm{AU}$. The solar spectrum we use is a synthesized spectrum from various 
sources [Berk et al., 2006, Kurucz, 1995], available at the MODTRAN web site http://rredc.nrel.gov/solar/spectra/am0/other_spectra.html.

The next step of our analysis is the determination of the error on the radiance. The derived error will be used in the best fit minimization of the reflectance spectra (see Section 3.4). At each wavelength, the radiance map (see Figure 2, panel a) is smoothed using a moving resistant mean in a $3 \times 3$ pixel box (see Figure 2. panel b). Outliers that vary by more than $2.5 \sigma$ are excluded. The smoothed radiance map is then subtracted from the original one, producing a background noise map (see Figure 2, panel c) where coma structures are no longer visible, except where the jets are particularly strong (e.g., jets originating from the lower edge of the larger lobe of the nucleus). For each pixel of the background noise map, the standard deviation of the mean of its neighboring pixels located in a 3-by-3 pixel box is computed and assumed to be the radiance error. A careful examination of panel (c) in Figure 2 allows us to conclude that this approach can be applied everywhere except for areas with coma structures that still remain in the background map. Avoiding these areas we take the mean uncertainty of the background at each wavelength (averaged over all pixels within the white boxes in panel c of Figure 2) as the uncertainty for all pixels in the image. This approach computes the intrinsic radiance error, dominated by warm bench background [for a detailed description of the sources of error in the HRI-IR instrument, the reader is referred to Klaasen et al., 2008, 2013]. The error ranges from a few percent at short wavelengths to a fraction of a percent at long wavelengths. The uncertainty in the absolute instrument calibration is $10 \%$ [Klaasen et al., 2013]. However, the spectral fitting analysis is performed without considering this $10 \%$ absolute error. 


\subsection{Dominant Spectral Features}

Figure 3 shows the comparison between two reflectance spectra extracted inside and outside the jet emerging from the small lobe of the nucleus facing the Sun as observed $7 \mathrm{~min}$ post-CA. Both spectra in Figure 3 are red sloped (increasing reflectance with increasing wavelength) from $1.2-2.5 \mu \mathrm{m}$. Specifically, the spectra extracted in boxes A (top panel of Figure 3) and B (bottom panel of Figure 3) of Figure 2 (panel a) have a spectral slope of 5.10 $\pm 0.08 \% / 0.1 \mu \mathrm{m}$ and $1.41 \pm 0.02 \% / 0.1 \mu \mathrm{m}$, respectively. In general, red slopes are well reproduced by refractory components (e.g., amorphous carbon) [Campins et al., 2006]. Beyond $2.5 \mu \mathrm{m}$, the spectrum is the sum of two components, one from scattered sunlight and one from thermal emission. Several emission features can also be seen in the reflectance spectra. These include emission from $\mathrm{H}_{2} \mathrm{O}$ vapor at $2.7 \mu \mathrm{m}, \mathrm{CO}_{2}$ at $4.3 \mu \mathrm{m}$, and organics in the $3.3-3.5 \mu \mathrm{m}$ region. These emission features will not be discussed further in this paper, as they are addressed by Feaga et al. [in prep] and Besse et al. [in prep]. While most areas in the coma do not show significant absorption features (Figure 3, top panel), the spectra extracted in the jet region (Figure 3, bottom panel) clearly display water ice absorption bands at 1.5, 2.0, and $3.0 \mu \mathrm{m}$. Water ice is blue in the near infrared, which explains the lower spectral slope of the water ice-rich spectrum with respect to the water ice-depleted one. The region sampled to display the water ice-rich spectrum in this paper (box B) is similar to that discussed by A'Hearn et al. [2011] in his Figure 5 (bottom right panel). Unlike box B, box A is displaced from the region sampled by A'Hearn et al. [2011] to display the water ice-depleted spectrum (see Section 3.4). 


\subsection{Modeling Analysis}

The flux from the coma in the wavelength range covered by the HRI-IR spectrometer is the sum of the scattered $\left(F_{\text {scat }}\right)$ and thermal $\left(F_{\text {thermal }}\right)$ components, the latter being dominant beyond $3.4 \mu \mathrm{m}$ (see dash-dot orange line in Figure 3).

\subsubsection{Scattered Component}

The scattered flux is calculated at each wavelength via

$$
F_{\text {scat }}(\lambda)=\frac{F_{\odot}(\lambda) p(\lambda) \Phi(\alpha) C}{\pi r^{2} \Delta^{2}}
$$

where $p(\lambda)$ is the geometric albedo of each single grain in the coma, $\Phi(\alpha)$ is the scattering phase function, $\Delta[\mathrm{m}]$ is the spacecraft-comet distance, and the scale factor $C\left[\mathrm{~m}^{2}\right]$ represents the total geometric cross section of the cometary grains within the slit. The contribution of the scattered flux to the reflectance, indicated as $R_{\text {scat }}$, is given by

$$
R_{\text {scat }}(\lambda)=f \Phi(\alpha) p(\lambda)
$$

where $f$ is the filling factor of the grains in the field of view (see Appendix A). The coma is a collection of many individual grains. In this analysis all grains are considered to be identical, each with geometric albedo $p(\lambda)$, computed by means of the Hapke [1993] radiative transfer model. We adopt the standard approach that a single grain is composed of independent scatterers, or particles. The qualitative spectroscopic analysis presented in Section 3.2 suggests the presence of two particle types: water ice and refractories. Two modes of mixing are possible: areal and intimate. Areal mixing (also called linear mixing) assumes that the different types of particles are spatially well separated and that a photon does not encounter different particle composition in one scattering event. In an intimate mixture, the 
particles are mixed homogeneously together in close proximity allowing for multiple scattering.

In the case of areal mixtures, the geometric albedo of each average grain consists of a linear combination of the geometric albedo of each component $p_{j}(j=$ $\mathrm{H}_{2} \mathrm{O}$-ice, refractories) weighted by its spatial extent:

$$
p(\lambda)=\sum_{j} F_{j} p_{j}(\lambda)
$$

where $F_{j}$ is the fraction of the area occupied by the $j$ th component, such that $\sum_{j} F_{j}=1$. In the case of isotropic scattering we have

$$
p_{j}(\lambda)=\frac{1}{2} r_{j}(\lambda)+\frac{1}{6} r_{j}^{2}(\lambda)
$$

where $r_{j}(\lambda)$ is the diffusive reflectance of the $j$ th component, which is a function of the particle single scattering albedo $w_{j}(\lambda)$ via

$$
r_{j}(\lambda)=\frac{1-\sqrt{1-w_{j}\left(n_{j}(\lambda), k_{j}(\lambda),<D_{j}>\right)}}{1+\sqrt{1-w_{j}\left(n_{j}(\lambda), k_{j}(\lambda),<D_{j}>\right)}} .
$$

The particle single scattering albedo describes the scattering properties of the material. It is computed by means of the Hapke [1993] equivalent-slab and exponential models for large $(X=\pi D / \lambda>>1$, where $\lambda$ is the wavelength of the incident radiation and $D$ is the particle diameter) irregular particles. The particle single scattering albedo is a function of the optical constants $n_{j}$ and $k_{j}$, the real and imaginary part of the refractive index, respectively, and $\left\langle D_{j}\right\rangle$, which represents the mean ray path length within the $j$ th-type of particle. In the case of spherical particles, $<D_{j}>\simeq 0.9 D_{j}$. However, if the particles are irregular with maximum dimension $D_{j}$, then $\left\langle D_{j}>\right.$ can be quite different than $D_{j}$, and in general will be smaller (i.e., they have smaller mean cross sections than the circumscribed sphere). We will from now on refer to $\left\langle D_{j}>\right.$ as particle diameter. 
In the case of intimate mixture, the averaging process is on the level of the scattering properties of the various types of particles in the mixture [Hapke, 1993]. The parameters that enter in the reflectance equation are averages of those of each particle type weighted by cross-sectional area (given by the fractional volume $V$ divided by the size of the grains $D$ ). We have the following equation for the geometric albedo:

$$
p(\lambda)=\frac{1}{2} r(\lambda)+\frac{1}{6} r^{2}(\lambda),
$$

where

$$
r(\lambda)=\frac{1-\sqrt{1-\bar{w}(\lambda)}}{1+\sqrt{1-\bar{w}(\lambda)}}
$$

with

$$
\bar{w}(\lambda)=\left[\sum_{j} \frac{V_{j}}{D_{j}} w_{j}\right]\left[\sum_{j} \frac{V_{j}}{D_{j}}\right]^{-1} .
$$

\subsubsection{Thermal Component}

We model the thermal flux density $F_{\text {thermal }}$ as

$$
F_{\text {thermal }}(\lambda)=\frac{\epsilon(\lambda) B\left(T_{c}, \lambda\right) C_{e}}{\Delta^{2}}
$$

where $\epsilon(\lambda)$ is the emissivity, $C_{e}$ is the emitting cross section, $T_{c}[\mathrm{~K}]$ is the coma temperature, and $B$ is the Planck function defined by the following equation

$$
B\left(T_{c}, \lambda\right)=\frac{2 h c^{2}}{\lambda^{5}}\left(e^{\frac{h c}{\lambda k_{b} T_{c}}}-1\right)^{-1}
$$

where $k_{b}$ is the Boltzmann constant, $h$ the Planck constant, and $c$ the speed of light. The contribution of the thermal flux to the reflectance, indicated as $R_{\text {thermal }}$, is given by

$$
R_{\text {thermal }}(\lambda)=\frac{\pi r^{2} \epsilon(\lambda) B\left(T_{c}, \lambda\right) f_{e}}{F_{\odot}}
$$

where $f_{e}$ is the filling factor of the grains. 


\subsection{Modeling Results}

In our analysis, to model the observed reflectance spectra, we first consider an areal mixture (the intimate mixing mode is analyzed in Section 3.7) of crystalline water ice ( $T=266 \mathrm{~K}$, optical constants from Warren and Brandt [2008]) with a dark and featureless refractory component (e.g., amorphous carbon, optical constants from Edoh [1983]). Because there are no absorption features detected in the near-infrared range other than those of water ice, the non-icy component cannot be unambiguously identified, and we adopt a material that reproduces the reddening characteristics. The variety of compounds that can be used is limited by the small number of materials whose optical constants have been determined over the range of wavelengths studied here, but silicates, organics (e.g., tholins), and amorphous carbon are possible candidates [Davies et al., 1997; Kawakita et al., 2004; Yang et al., 2009]. Amorphous carbon (AC) was selected because it is featureless and is the lowest albedo reddening agent. Its characteristics, compared to the other materials, imply that our results will provide an upper limit on the column density of refractories.

We assume $\epsilon(\lambda)=1$ and neglect $\Phi(\alpha)$, given the small variations of phase angle ranging between $90^{\circ}$ and $94^{\circ}$ for data acquired within $\sim 10 \mathrm{hr}$ post-CA. Also, due to the heliocentric distance of comet Hartley 2 (1.06 AU) and the small field of view $(\sim 500 \times 500 \mu \mathrm{rad})$, the phase angle can be considered constant for all grains along the line of sight of the spacecraft. Furthermore, the proper treatment would consist of considering a dust phase function in water ice-depleted regions and a phase function for aggregates of water ice grains, or comae composed of water ice and dust in water ice-enriched regions. However, while the former is approximated by Schleicher and Bair [2011], the latter has not been estimated yet. Thus, 
we prefer to leave the phase function as 1.0 for both components, rather than make an assumption on one or both of these populations. Therefore, the free parameters in our model are the water ice-to-dust fraction $\left(F_{\mathrm{H}_{2} \mathrm{O}}\right)$, the coma temperature $T_{c}$, and the scattering $(f)$ and emitting $\left(f_{e}\right)$ filling factors. They are iteratively modified by means of a $\chi^{2}$ minimization algorithm (Levenberg-Marquardt leastsquares minimization) until the best fit to the observations is achieved. To exclude the locations where emission bands occur, the optimization is performed only from $1.30-1.80 \mu \mathrm{m}, 1.95-2.50 \mu \mathrm{m}, 2.91-3.20 \mu \mathrm{m}, 3.60-4.10 \mu \mathrm{m}$, and 4.45 $-4.70 \mu \mathrm{m}$. The particle diameters of both components in the mixture $\left(<D_{\mathrm{H}_{2} \mathrm{O}}\right\rangle$ and $\left\langle D_{A C}\right\rangle$ ) are assumed equal to $1 \mu \mathrm{m}$ (see next Section). The modeling is applied to each pixel and the results are averaged in a 3-by-3 pixel box. Figures 3 and 4 show the comparison between the observed reflectance spectra (triangles) extracted in boxes A and B from $1.2-4.8 \mu \mathrm{m}$ and the best-fit model at same spectral resolution as the HRI-IR observations. The synthetic spectra match all the main features of the observed spectra from $1.2-4.8 \mu \mathrm{m}$. The parameters of the best-fit models shown in Figures 3 and 4 are listed in Table 3 (see Model 1, Box A and B). Specifically, the free parameters listed in Table 3 are the 3-by-3 pixel box averages of the modeled results together with the standard error of the mean. The same approach is applied to the reduced $\chi_{\text {red }}^{2}$. The latter in case of Model 1 computed in boxes A and B is 10 and 16, respectively, for a number of degrees of freedom equal to 230. We attribute the high value of $\chi_{\text {red }}^{2}$ to a possible underestimation of the error and to the high quality of the data with respect to the optical constants. Furthermore, the possible presence of yet unidentified subtle emission bands in wavelength regions where the modeling is computed may raise the value of $\chi_{\text {red }}^{2}$. Additionally, the combination of our simple model and the 
chosen set of optical constants is not expected to fit the spectra exactly, given the many complexities of cometary comae.

The coma temperatures, $T_{c}$, derived from the modeling in both water ice-rich and water ice-depleted areas are similar. This indicates that the thermal component of the spectra is not sensitive to the water ice content and that $T_{c}$, around 300 $\mathrm{K}$, represents the effective dust temperature. The scattering $(f)$ and emitting $\left(f_{e}\right)$ filling factors differ by a factor of 2.0 in water ice-depleted areas (Box A, Table 3). This ratio would be increased to a factor of 3.4, if we were to adopt the comet dust phase function of Schleicher and Bair [2011]. However, our $f_{e}$ is dependent on the effective temperature of the spectrum, which may not be the true temperature of the thermally emitting grains, the latter being the more relevant quantity. Moreover, the thermal emission spectrum may be the result of the superposition of many different grains with different radiative temperatures. Finally, the grains that dominate the scattered light are not necessarily the same grains that dominate the thermal emission at these wavelengths. Altogether, we report our best-fit values, but do not interpret them any further.

Modeling of the 3- $\mu \mathrm{m}$ region for those spectra displaying water ice absorptions requires special care. The long wavelength shoulder of the $2.7-\mu \mathrm{m}$ water vapor emission is partially superimposed on the water ice absorption. Therefore, it is difficult to extract information about the width of the 3- $\mu$ m water ice absorption. A correct estimate of the water ice temperature and grain size relies on the knowledge of the width of the 3- $\mu \mathrm{m}$ water ice absorption as discussed in Sections 3.5 and 3.6. As a means of evaluating our model, we compare the width of the water gas emission band in the emissivity spectra extracted from both water ice-rich and water ice-depleted regions. For this purpose we avoid areas in the cometary 
coma which display the densest enrichment of water gas (e.g., the water gas plume near the waist of the nucleus discussed by A'Hearn et al. [2011]), where optical depth effects are expected and may change the shape and width of the water gas emission. Figure 5, panels (a) and (b), show the same spectra discussed above, but converted to radiance to allow inspection of the emission bands. Panels (c) and (d) show the resultant emissivity spectra obtained by subtracting the model from the observed radiance spectra. The emissivity spectra outside the emission bands are close to zero, indicating that the continuum is adequately modeled as well as the width and the depth of the 1.5- and 2.0- $\mu \mathrm{m}$ water ice absorptions. The widths of the water gas emission extracted in both depleted and rich water ice regions are comparable extending from 2.55 to $2.90 \mu \mathrm{m}$, indicating that the $3.0-\mu \mathrm{m}$ water ice absorption band has been modeled correctly, thus validating our approach.

\subsection{Water Ice and Refractory Grains: Particle Size Effects}

Figure 6 shows the comparison between the water ice-depleted spectrum observed 7 min post-CA and the synthetic spectrum obtained from an areal mixture of water ice $\left.\left(<D_{H_{2} O}\right\rangle=1 \mu \mathrm{m}\right)$ and amorphous carbon, with $<D_{A C}>$ equal to $1 \mu \mathrm{m}, 2 \mu \mathrm{m}$, and $5 \mu \mathrm{m}$. The modeling results are listed in Table 3 (see Box A, Model 1, 2, and 3, respectively). The contribution of water ice is always less than $0.1 \%$, as expected since the observed spectrum is featureless. The modeling analysis of the water ice-depleted spectra is therefore only sensitive to the characteristics of the refractories, allowing us to investigate the particle size effects of the refractory grains. The observed spectral slope is compatible with amorphous carbon on the order of $1 \mu \mathrm{m}$. However, because amorphous carbon is strongly absorbing, its calculated reflectance is not strongly dependent on grain size, as indicated by each model's $\chi_{\text {red }}^{2}$ (Table 3 . The results from this analysis support 
holding the refractory grain size equal to $1 \mu \mathrm{m}$ in the next paragraph.

Figure 7 shows the comparison between the water ice-rich reflectance spectrum of box B in Figure 2 and the synthetic spectrum obtained from an areal mixture of amorphous carbon $\left(<D_{A C}>=1 \mu \mathrm{m}\right)$ and water ice, with $\left\langle D_{H_{2} O}>\right.$ as a free parameter. Results are given in Table 3 (Box B, Model 2). The best value for $\left\langle D_{\mathrm{H}_{2} \mathrm{O}}\right\rangle$ (red line) is on the order of $1 \mu \mathrm{m}(0.82 \mu \mathrm{m})$ and the goodness of fit is the same as in the case of Model 1 obtained with a fixed value of $1 \mu \mathrm{m}$ for $<D_{\mathrm{H}_{2} \mathrm{O}}>$. Water ice particles on the order of $2 \mu \mathrm{m}$ in size provide a similarly good fit to the data (see blue line, Table 3, Box B, Model 3). The purple, green, and brown lines in Figure 7 represent the synthetic spectra for $\left\langle D_{\mathrm{H}_{2} \mathrm{O}}\right\rangle$ equal to 5,10 , and $100 \mu \mathrm{m}$, respectively. The fits in these cases are considerably worse, as also indicated by their $\chi_{\text {red }}^{2}$. This is because the width and relative strength of the water ice absorption bands at 1.5, 2.0, and 3.0 $\mu \mathrm{m}$ are strongly dependent on the water ice grain size, as shown in the inserted panel of Figure 7 . We therefore conclude that the three absorption features observed at 1.5, 2.0, and $3.0 \mu \mathrm{m}$ are consistent in bandwidth and strength with the presence of water ice grains of size less than $5 \mu \mathrm{m}$ in the coma. We acknowledge that even if $\left\langle D_{\mathrm{H}_{2} \mathrm{O}}\right\rangle$ is generally smaller than $D_{\mathrm{H}_{2} \mathrm{O}}$, which is what appears in the $X=\pi D / \lambda>>1$ relation, we are not necessarily comfortably within the Hapke [1993] geometric optics regime.

\subsection{Phase and Temperature of the Water Ice}

Laboratory measurements show that infrared water ice absorption bands change position and shape as a function of phase (crystalline or amorphous) and temperature [Grundy and Schmitt, 1998, Mastrapa et al., 2008]. Figure 8 illustrates the variations in the modeling of the Hartley 2 water ice-rich spectrum for different phases and temperatures of the water ice. Specifically, we compare the spectral 
modeling obtained using Mastrapa et al. [2009] optical constants of amorphous (Ia) and crystalline (Ic) water ice at $120 \mathrm{~K}$ (purple and yellow lines in Figure 8 and Models 7 and 8 in Table 3, respectively) and Warren and Brandt [2008] optical constants of crystalline water ice at $266 \mathrm{~K}$ (red line in Figure 8, Model 1 Box B in Table 3). The inserted panel in Figure 8 shows the comparison between reflectance spectra of $100 \%$ water ice on the order of $1 \mu \mathrm{m}$ computed using these three sets of optical constants.

The $1.65-\mu \mathrm{m}$ water ice absorption band is strongly dependent on phase and temperature (see Figure 8, inserted panel). This band is evident in reflectance spectra of cold crystalline ice [Grundy and Schmitt, 1998], but less prominent or absent in amorphous ice. Crystalline ice indicates formation temperatures in excess of $130 \mathrm{~K}$, the critical temperature for transformation from amorphous to crystalline ice [Grundy and Schmitt, 1998; Jewitt and Luu, 2004]. However, the $1.65-\mu \mathrm{m}$ band reduces in strength as temperature increases, and it almost disappears in crystalline ice $\mathrm{T} \geq 230 \mathrm{~K}$ [Grundy and Schmitt, 1998; Mastrapa et al., 2008]. We do not detect the 1.65- $\mu \mathrm{m}$ absorption band in Hartley 2 spectra, so we can not distinguish between amorphous water ice and "warm" crystalline water ice.

Another band strongly dependent on the water ice deposition temperature is the 3- $\mu \mathrm{m}$ band. As observed by Mastrapa et al. [2009], the band at $3 \mu \mathrm{m}$ is stronger and shifted to longer wavelengths in crystalline water ice compared to that of amorphous water ice. The differences between the Mastrapa et al. [2009] and Warren and Brandt [2008] optical constants around $3 \mu \mathrm{m}$, discussed by Mastrapa et al. [2009], are possibly due to differences in laboratory methods (see Figure 8, inserted panel). Qualitatively, the Warren and Brandt [2008] data set 
provides a better fit to the data around $3.5 \mu \mathrm{m}$. However, comparing the modeling results reported in Table 3, we can not determine the phase (amorphous versus crystalline) and temperature of the water ice. A limiting factor is the spectral differences seen in laboratory measurements between amorphous and crystalline ice around $3 \mu \mathrm{m}$ fall in regions overlapping with gas emissions, which are not used to optimize our modeling.

\subsection{Well Separated or Intimate Mixing}

Using a linear mixing model, the Hartley 2 data are consistent with $1-\mu \mathrm{m}$ diameter water ice particles. The resulting model, shown in Figure 7, matches the ice absorptions present in the data at 1.5, 2.0 and 3.0 $\mu \mathrm{m}$ (Section 3.5). Figure 9 shows that no particle size of water ice is consistent with the observations when intimately mixed with refractory materials. The results displayed in Figure 9 are from models of water ice and amorphous carbon $\left(<D_{A C}>=1 \mu \mathrm{m}\right)$ using optical constants from Warren and Brandt [2008] and Edoh [1983], respectively. The free parameters in our model are the water ice-to-dust ratio $\left(F_{\mathrm{H}_{2} \mathrm{O}}\right)$, the coma temperature $T_{c}$, and the scattering $(f)$ and emitting $\left(f_{e}\right)$ filling factors. Large water ice particle sizes $($ e.g., $10 \mu \mathrm{m})$ match the ice absorption near $2 \mu \mathrm{m}$, but the $3-\mu \mathrm{m}$ region is modeled poorly. When using smaller particle sizes, the models have weak or absent $2-\mu \mathrm{m}$ bands with respect to the observations and 3- $\mu \mathrm{m}$ absorptions that are narrower than the data. We can therefore conclude that intimate mixing can be excluded. The fact that the observed spectra, particularly the strengths and shapes of the three absorption bands of water ice, are well reproduced by an areal mixture of water ice and refractories implies that water ice in the coma of Hartley 2 is relatively pure. 


\section{Spatial distribution of the water ice and refractories}

The modeling analysis described in Sections 3.3 and 3.4 (areal mixture of water ice and amorphous carbon) is applied to each pixel of the 5006000 and 5007002 scans. Over the entire scans, no variation in particle size was detected. Thus, for each scan we compute the water ice-to-dust ratio map $\left(F_{\mathrm{H}_{2} \mathrm{O}}\right)$ and the scattering filling factor map $(f)$, assuming a grain size of $1 \mu \mathrm{m}$ for water ice and dust. We compute the water ice and dust column density as

$$
N_{j} \leq \frac{F_{j} f}{\pi\left(<D_{j}>/ 2\right)^{2}}
$$

where $j$ refers to water ice or dust (represented by amorphous carbon). In the case of dust, we have $F_{j}=1-F_{\mathrm{H}_{2} \mathrm{O}}$. The column density of the water ice and dust, expressed in number of particles per square meter, is shown in Figure 10. These maps have been smoothed applying a resistant mean (2.5 $\sigma$ threshold) in a 3-by-3 pixel box. The column density, $N_{j}$, computed via Equation 13 , is an upper limit,

given that $\left\langle D_{j}>\right.$ represents the diameter of the particles within a grain (see Section 3.3, not the total grain aggregate diameter.

Inspection of panel (B) of Figure 10 reveals that water ice is not uniformly distributed in the innermost coma of Hartley 2. It is possible to observe the presence of water ice-enriched $\left(\geq 6 \times 10^{6}\right.$ particles $/ \mathrm{m}^{2}$, up to values of $\sim 2.5 \times 10^{9}$ particles $/ \mathrm{m}^{2}$ near the limb of the small lobe of the nucleus facing the Sun) and water ice-depleted $\left(\leq 10^{6}\right.$ particles $\left./ \mathrm{m}^{2}\right)$ regions. Water ice is found in 5 out of the 6 strongest jets ( $J_{1}$ through $J_{5}$, as labeled in panel A). The only jet not enriched in water ice is the one off the side of the large lobe facing the Sun and labeled in panel (A) as $\mathbf{J}_{6}$. The comparison between the HRI-VIS context image acquired 7 min post-CA (panel A) and the corresponding water ice column density map 
(panel B) suggests a strong correlation between the brightness distribution in the coma of Hartley 2 and the water ice abundance, as seen by A'Hearn et al. [2011]. Unlike water ice, dust is everywhere in the innermost coma of Hartley 2, with an enhancement in the jets (Figure 10, panel C). Because the water ice and dust column densities differ by a factor of 10 , we can conclude that the innermost coma of Hartley 2 is dominated by dust. Similar conclusions are obtained for the water ice and dust column density maps derived from the 5007002 scan acquired 23 min post-CA, although the spatial resolution is lower than scan 5006000 (Figure 10, right column). As observed in Section 3.4, amorphous carbon is one of the lowest albedo reddening agents, therefore the dust column density provided here represents an upper limit. However, even if astronomical silicates are used as a refractory component instead of amorphous carbon (not shown), the innermost coma of Hartley 2 is still dominated by dust.

\subsection{Driver of activity}

Figure 11 shows the spatial distribution of the water ice and dust in the innermost coma of Hartley 2 compared with that of gaseous water and $\mathrm{CO}_{2}$, as observed 7 min (ID scan 5006000, left column) and 23 min (ID scan 5007002, right column) post-CA. The gaseous-water and $\mathrm{CO}_{2}$ maps have been obtained computing the total of the emissivity spectral cubes (see Section 3.4. Figure 5) in the 2.55 $-2.90 \mu \mathrm{m}$ and $4.15-4.45 \mu \mathrm{m}$ wavelength ranges, respectively. The maps have been smoothed by averaging in a running $3 \times 3$ pixel box. The water vapor and $\mathrm{CO}_{2}$ spatial distributions are discussed here only qualitatively, since a quantitative analysis is presented by A'Hearn et al. [2011] and Feaga et al. [in prep]. The maps in Figure 11 show a water vapor-rich region extending roughly perpendicular to the waist of the nucleus (panels $\mathrm{A}$ and $\mathrm{E}$ ). This region has relatively little $\mathrm{CO}_{2}$ 
(panels $\mathrm{B}$ and $\mathrm{F}$ ) and no water ice. The $\mathrm{CO}_{2}$ is concentrated in jets, with a particular enhancement in the region off the end of the smaller lobe of the nucleus facing the Sun (jet $\mathrm{J}_{1}$ in panel A of Figure 10 ). The spatial distribution of the water ice grains (panels D and $\mathrm{H}$ in Figure 11) and dust (panels $\mathrm{C}$ and $\mathrm{G}$ in Figure 11), very different than the water vapor distribution, is strongly correlated with the $\mathrm{CO}_{2}$ rich jets (most strongly in the jet $\mathrm{J}_{1}$ ). This correlation validates the suggestion put forth by A'Hearn et al. [2011] that $\mathrm{CO}_{2}$, rather than water gas, drags water ice grains into the coma as it leaves the nucleus. Our improved spatial distribution maps of water ice and $\mathrm{CO}_{2}$, including additional temporal coverage and maps of the dust distribution, reinforce the importance of $\mathrm{CO}_{2}$ in Hartley 2's activity not only for the water ice but also for the dust.

\subsection{Radial Distributions of Water Ice and Dust}

We focus on the variations of the water ice and dust column densities with nucleocenteric distance $\rho$ along the jets $\mathrm{J}_{1}$ and $\mathrm{J}_{4}$ observed at CA+7 min (see panel A of Figure 10, with the goal of shedding light on the mechanisms that govern the inner coma of Hartley 2. Our analysis is conducted in the $\mathbf{J}_{1}$ and $\mathbf{J}_{4}$ jets since they are the brightest. We compute the average column densities in annular sectors with radii $\rho$ and $\rho+\mathrm{d} \rho$, with $\mathrm{d} \rho=0.1 \mathrm{~km}$, and azimuthal angles defined by the dash-dot green lines in Figure 10, panel (A), which enclose the area of the jets $\mathbf{J}_{1}$ and $\mathbf{J}_{4}$. For the purposes of calculation, we assume the source of the jets is the center of the surface of curvature at which the material leaves the nucleus. Therefore, $\rho$ specifically represents the distance from the center of curvature. The variation of column density with $\rho$ can suggest whether a particular component sublimes, fragments, is accelerated, or is in a constant outflow. For a normal cometary coma described by a simple outflow, the column density would follow 
a $1 / \rho$ radial profile. Jets and fans do not change this trend. Figure 12 shows the column density of dust and water ice as a function of $\rho$ in jets $\mathbf{J}_{1}$ off the small lobe facing the Sun and $\mathbf{J}_{4}$ off the lower edge of the larger lobe in the anti-sunward direction. Both dust profiles can be well fitted by the function

$$
f(\rho)=\alpha \rho^{k}
$$

The modeling of the dust profiles is presented in Figure 12 and the values of $\alpha$ and $k$ are given in Table 4 . Since $k$ is close to -1 , we conclude that the dust presents a constant outflow profile. Water ice presents a steeper profile than $1 / \rho$, which suggests that water ice behaves differently than the dust and possibly that it sublimes very close to the nucleus in the coma. A steeper profile than $1 / \rho$ could also be explained in terms of water ice particles being accelerated. However, there is no reason for which the dust would not be accelerated as well. We therefore favor the sublimation scenario. Following the approach of Tozzi et al. [2004], we satisfactorily fit the water ice profiles with an exponential function

$$
f(\rho)=\frac{\beta}{\rho} e^{-\frac{\rho-\rho^{\prime}}{L}}
$$

where $\rho^{\prime}$ is the distance from the center of curvature to the surface and $L$ is the scale-length. This relationship reproduces the observed profiles (see dashed line in Figure 12). The values for $\beta, \rho^{\prime}$, and $L$, used to generate the best fit are reported in Table 4 . Given that the jet $\mathrm{J}_{1}$ is propagating along the sunward direction, nearly perpendicular to the line of sight, viewing geometry effects are, to a first approximation, negligible. This does not appear to be the case for the jet $\mathrm{J}_{4}$. The scale-length of the subliming water ice (636 $\mathrm{m}$ in the $\mathrm{J}_{1} \mathrm{jet}$ ) is compatible with lifetimes of $1-\mu \mathrm{m}$ pure water ice grains at $1 \mathrm{AU}[\sim 1 \mathrm{~h}$, corresponding to three times the $e$-folding time; Hanner, 1981] for velocities on the order of $0.5 \mathrm{~m} / \mathrm{s}$. 


\subsection{Water Ice Mass Production Rate}

The modeling of the water ice column density radial profile presented in the previous section can be used to estimate the water ice mass production rate. For the same arguments reported above, we focus on the jet off the small lobe of the nucleus facing the Sun (jet $\mathbf{J}_{1}$ ). The production rate $Q$ of a material with a $\Gamma / \rho$ column density profile within a jet of angular size $\theta$ is given by:

$$
Q=\frac{2 \pi \Gamma v\left(1-\cos \frac{\theta}{2}\right)}{\theta}
$$

where $v$ represents the outflow speed. With Equations 15 and 16, we can compute the water ice production rate. Letting $v=0.5 \mathrm{~m} / \mathrm{s}$ and $\Gamma=\beta$, we obtain in the jet $\mathbf{J}_{1}\left(\theta=54^{\circ}\right)$ a water ice production rate $Q_{H_{2} O-i c e}$ of $9 \times 10^{11} \pm 2 \times 10^{10}$ particles/s, which corresponds, for $1-\mu \mathrm{m}$ particles with density of $1 \mathrm{~g} \mathrm{~cm}^{-3}$, to a mass production rate of $\dot{M}=0.00046 \pm 0.00001 \mathrm{~kg} / \mathrm{s}$. The $2 \%$ error on $Q_{\mathrm{H}_{2} \mathrm{O}-i c e}$ and $\dot{M}$, has been computed considering the errors on $\beta$ and $L$, reported in Table 4 . However, according to our model the $1-\mu \mathrm{m}$ grains are in aggregates, and as such, their interiors may be shielded from our view. If so, and if the interiors are also comprised of water ice, our derived mass production rate is necessarily a lower limit.

\section{Discussion}

Comet Hartley 2 is a hyperactive comet with an active fraction near $1.7-2.5$ [Kelley et al., 2013]. The presence of an icy grain halo, suggested by Lisse et al. [2009], to account for the high water production rate of comet Hartley 2 seems plausible, but difficult to justify quantitatively. Two relevant particle populations have been detected so far in the coma of Hartley 2: (1) $1-\mu \mathrm{m}$ water ice grains 
in the inner few kilometers of the Hartley 2 coma (this paper), and (2) distinct, isolated grains that in visible images envelope the nucleus of Hartley 2 up to 40 $\mathrm{km}$, and estimated by their brightness to be centimeter-sized ( $1 \mathrm{~cm}$ in radius) or larger and possibly made of ice (density of $0.1 \mathrm{~g} / \mathrm{cm}^{3}$ ) [A'Hearn et al. 2011; Kelley et al., 2013]. If the latter particles are indeed icy, both will contribute to the total water production rate $\left[\right.$ e.g., $10^{28} \mathrm{molec} / \mathrm{s} \simeq 299 \mathrm{~kg} / \mathrm{s}$ A'Hearn et al. 2011] up to $2 \times 10^{-4} \%$ (upper limit estimated considering the $2 \%$ error on $\dot{M}$ ) and $0.5 \%$ [Kelley et al., 2013], respectively. Therefore, the 1- $\mu \mathrm{m}$ water ice particles and the $\mathrm{cm}$ particles observed in the visible images do not appear to be the source of the comet's enhanced water production. These observations, collectively, raise interesting questions on the relationship between the $1-\mu \mathrm{m}$ and $\mathrm{cm}$ size particles and the source of the hyperactivity of comet Hartley 2.

The estimated velocity consistent with our derived scale-lengths, $0.5 \mathrm{~m} / \mathrm{s}$, is much lower than the expansion velocities expected for isolated 1- $\mu \mathrm{m}$ water ice grains, the latter being on the order of $600 \mathrm{~m} / \mathrm{s}$ at $1 \mathrm{AU}$ [Hanner, 1981; Whipple, 1951]. This incongruency suggests that the water ice is in the form of large aggregates (millimeter to centimeter size), consistent with the applied spectroscopic modeling and the low inferred velocities. Our suggestion is based on the modeling by Fougere et al. [2013], who predict velocities up to $100 \mathrm{~m} / \mathrm{s}$ and $2 \mathrm{~m} / \mathrm{s}$ for grains of $1 \mu \mathrm{m}$ and $5 \mathrm{~mm}$, respectively. If we consider the case of large aggregates, e.g., particles on the order of $1 \mathrm{~cm}$ in radius, with density of $0.1 \mathrm{~g} \mathrm{~cm}^{-3}$ and our derived velocities of $0.5 \mathrm{~m} / \mathrm{s}$, we estimate the aggregate contribution to the total water production rate to be $0.3 \%$, in agreement with the favorable scenario put forth by Kelley et al. [2013]. This suggests that the large chunks observed in the visible are most likely made of $1-\mu \mathrm{m}$ water ice particles. One caveat important to 
mention is that the expansion velocity value of $0.5 \mathrm{~m} / \mathrm{s}$, has been measured considering the $e$-folding time of $1-\mu \mathrm{m}$ water ice particles at $1 \mathrm{AU}$ reported by Hanner [1981], which refers to single particles and not aggregates. Further studies are needed to improve our understanding of the lifetimes of water ice aggregates.

The conclusion that the 1-cm large chunks detected in the visible data are aggregates of $1-\mu \mathrm{m}$ water ice particles is supported by our estimate of the total water ice cross section, given by $f F_{\mathrm{H}_{2} \mathrm{O}-i c e} \sigma$, being $\sigma$ the geometric cross section of one pixel, and equal to $0.001 \mathrm{~km}^{2}$ in a field of view of $4.7 \times 2.7 \mathrm{~km}^{2}$ as measured from the HRI-IR data acquired $7 \mathrm{~min}$ and $23 \mathrm{~min}$ post-CA. This value is in agreement with the total icy particle cross section estimated from the MRI observations of the large chunks being in the range of $0.0004-0.003 \mathrm{~km}^{2}$ within $20.6 \mathrm{~km}$ from the nucleus [Kelley et al., 2013].

Even if, at first order approximation, we are able to reconcile our analysis with the MRI-and HRI-VIS observations of the large chunks in the coma of Hartley 2, we still find some discrepancies when comparing our results with other observations. The measured scale-length of $\sim 600 \mathrm{~m}$ implies that not much water ice would survive up to $40 \mathrm{~km}$ from the nucleus, in contradiction with the MRI-VIS observations of the large chunks up to this distance [Kelley et al. 2013]. If water ice particles were entrained in the $\mathrm{CO}_{2}$ gas flow, as suggested by the spatial correlation between the water ice and $\mathrm{CO}_{2}$ and assumed to be three orders of magnitude faster, then ice particles could be observed at larger distances from the nucleus. However, not only ice but also dust would be carried by the $\mathrm{CO}_{2}$ and no evidence of acceleration is seen in the dust profiles. Our observations are still unable to solve the mystery about the main source of water in Hartley 2's coma. Indeed, while we can rule out the case of isolated 1- $\mu \mathrm{m}$ water ice grains as the 
main contributor to the total water production rate, the case of aggregates of 1$\mu \mathrm{m}$ remains open. To confirm or reject the prediction by Fougere et al. [2013] that the sublimating icy grains in the Hartley 2's coma release $\sim 77 \%$ of the total water particles, advanced studies are needed to further understand how water ice aggregates sublime and constrain the unknown size and density of the aggregates. We have so far considered as size and density of these aggregates the values suggested by Kelley et al. [2013] to describe the large chunks observed in the MRIand HRI-VIS data. However, a final resolution on the relationship between 1- $\mu \mathrm{m}$ water ice grains detected in near-IR scans and the large chunks described by Kelley et al. [2013] is still open. We have recently identified in near-IR scans point sources corresponding to the large chunks observed in HRI- and MRI-VIS data. The analysis of the spectroscopic properties of these point sources, which will be subject of a future work, will shed light on the composition of the large chunks observed in the visible data together with their relationship with the 1 - $\mu \mathrm{m}$ water ice grains detected in the near-IR scans, and maybe help us solving the mystery about the main source of water in Hartley 2's coma.

This paper focused on producing the best dataset yet available for studying a comet's innermost coma. We also included some very basic modeling, with several of simplifying assumptions, to get some preliminary results regarding the comet's environment. The discrepancies that we find between these results and other observations show that these simple models are clearly insufficient for explaining the data, and detailed models must be developed to produce a more comprehensive explanation of the observations. 


\section{Conclusions}

We have analyzed DI HRI-IR spatially resolved scans of comet Hartley 2, collected $7 \mathrm{~min}$ (ID 5006000) and $23 \mathrm{~min}$ (ID 5007002) after closest approach, when the comet was at a heliocentric distance of $1.06 \mathrm{AU}$ and with spatial scales at mid-scan of $55 \mathrm{~m} /$ pix and $173 \mathrm{~m} /$ pix, respectively. The reflectance spectra show clear absorption bands near 1.5, 2.0, and $3.0 \mu \mathrm{m}$, consistent in central wavelength, band width, and band shape with the presence of water ice grains in the coma. Using Hapke's radiative transfer model we find that the spectra are best fit by an areal mixture of a featureless, highly absorbing, refractory component and water ice grains with size on the order of $1 \mu \mathrm{m}$. Intimate mixtures of the same materials cannot reproduce the spectra; a strong indication that the grains of water ice are relatively pure, i.e., relatively free of refractory material. In addition, the measured temperature of the grains is on the order of $300 \mathrm{~K}$, which is inconsistent with water ice persisting long enough to be observed, implying that the thermal emission is purely from the refractory component and that the refractory grains are not in thermal contact with the grains of water ice.

In the innermost coma, within a nuclear radius of the surface, dust dominates over water ice by a factor 10 , with column densities of $10^{9}$ and $10^{10}$ particles $/ \mathrm{m}^{2}$ of water ice and dust, respectively. The water ice and the dust are not uniformly distributed - their spatial distribution is strongly correlated with the presence of $\mathrm{CO}_{2}$ emission but very different from the distribution of water vapor emission, indicating that the $\mathrm{CO}_{2}$ plays a key role in Hartley 2's activity for both water ice and dust and that the "waist jet" of gaseous $\mathrm{H}_{2} \mathrm{O}$ arises from a different process than most of the outgassing. The radial profiles of the refractory dust follow a $1 / \rho$ profile, consistent with uniform outflow, while the radial profiles of water ice 
are much steeper. The differences between the profiles could be consistent with acceleration of the water ice but not the dust. However, it is more likely that the steeper profiles for ice are due to water ice sublimation. The lifetime of water ice grains at $1 \mathrm{AU}$ [Hanner, 1981] suggests that the expansion velocity of the water ice grains is of order $0.5 \mathrm{~m} / \mathrm{s}$. This in turn suggests that the $1-\mu \mathrm{m}$ grains seen in the spectra are the components of more massive aggregates, which could imply shielding of some grains even in the optically thin coma and that the column densities of water ice are higher than deduced from our modeling.

One can compare the icy grains in comet Hartley 2, a hyperactive comet from which icy grains are apparently dragged out continuously by gaseous $\mathrm{CO}_{2}$, with the icy grains mechanically excavated from 10-20-m depths in the low-activity comet Tempel 1 [Sunshine et al., 2007], and with the icy grains excavated by the infrequent but large natural outbursts of comet Holmes [Yang et al., 2009]. In all three cases, the icy grains are relatively pure and dominated by particles of order $1 \mu \mathrm{m}$ in size. Taken together this suggests that in most comets the ice in the interior of the nuclei is in the form of aggregates of relatively pure ice and that intimate mixtures of ice and refractories are rare. Furthermore, this implies that nuclei are commonly very porous, as suggested by the few bulk densities that have been determined and that aggregation models [e.g., Greenberg and Li, 1999] that call for grains with refractory cores, organic mantles, and icy crusts are generally inappropriate for comets.

\section{Acknowledgments}

The authors thank Dennis Bodewits and Dennis Wellnitz for useful discussions and the anonymous referees for comments that helped improving the quality of 
this paper.

This work was funded by NASA, through the Discovery Program, via contract NNM07AA99C to the University of Maryland and task order NMO711002 to the Jet Propulsion Laboratory.

\section{References}

A'Hearn, M. F., 2011. Comets as Building Blocks. Annual Review of Astronomy and Astrophysics 49, 281-299.

A’Hearn, M. F., et al., 2011. EPOXI at Comet Hartley 2. Science 332, 1396-.

A'Hearn, M. F., et al., 2005. Deep Impact: Excavating Comet Tempel 1. Science $310,258-264$.

A'Hearn, M. F., et al., 2012. Cometary Volatiles and the Origin of Comets. The Astrophysical Journal 758, 29.

Belton, M. J. S., et al., 2013. The complex spin state of 103P/Hartley 2: Kinematics and orientation in space. Icarus 222, 595-609.

Belton, M. J. S., et al., 2007. The internal structure of Jupiter family cometary nuclei from Deep Impact observations: The talps or layered pile model. Icarus $187,332-344$.

Berk, A., et al., 2006. MODTRAN5: 2006 update. In: Society of Photo-Optical Instrumentation Engineers (SPIE) Conference Series. Vol. 6233 of Society of Photo-Optical Instrumentation Engineers (SPIE) Conference Series.

Besse, S., et al., in prep. 
Bockelée-Morvan, D., Crovisier, J., Mumma, M. J., Weaver, H. A., 2004. The composition of cometary volatiles. pp. 391-423.

Bockelée-Morvan, D., Rickman, H., 1997. C/1995 O1 (Hale-Bopp): Gas Production Curves And Their Interpretation. Earth Moon and Planets 79, 55-77.

Campins, H., et al., 2006. Nuclear Spectra of Comet 162P/Siding Spring (2004 TU12). The Astronomical Journal 132, 1346-1353.

Davies, J. K., et al., 1997. The Detection of Water Ice in Comet Hale-Bopp. Icarus $127,238-245$.

Donn, B., Daniels, P. A., Hughes, D. W., 1985. On the Structure of the Cometary Nucleus. In: Bulletin of the American Astronomical Society. Vol. 17 of Bulletin of the American Astronomical Society. p. 520.

Donn, B., Hughes, D., 1986. A fractal model of a cometary nucleus formed by random accretion. In: Battrick, B., Rolfe, E. J., Reinhard, R. (Eds.), ESLAB Symposium on the Exploration of Halley's Comet. Vol. 250 of ESA Special Publication. pp. 523-524.

Edoh, O., 1983. Optical properties of carbon from the far infrared to the far ultraviolet. PhD Thesis, University of Arizona.

Feaga, L. M., A’Hearn, M. F., Sunshine, J. M., Groussin, O., Farnham, T. L., 2007. Asymmetries in the distribution of $\mathrm{H}_{2} \mathrm{O}$ and $\mathrm{CO}_{2}$ in the inner coma of Comet 9P/Tempel 1 as observed by Deep Impact. Icarus 190, 345-356.

Feaga, L. M., et al., in prep. 
Fougere, N., Combi, M. R., Rubin, M., Tenishev, V., 2013. Modeling the heterogeneous ice and gas coma of Comet 103P/Hartley 2. Icarus 225, 688-702.

Gombosi, T. I., Houpis, H. L. F., 1986. An icy-glue model of cometary nuclei. Nature 324, 43.

Greenberg, J. M., 1998. Making a comet nucleus. Astronomy and Astrophysics $330,375-380$.

Greenberg, J. M., Li, A., 1999. Morphological Structure and Chemical Composition of Cometary Nuclei and Dust. Space Science Reviews 90, 149-161.

Grundy, W. M., Schmitt, B., 1998. The temperature-dependent near-infrared absorption spectrum of hexagonal $\mathrm{H}_{2} \mathrm{O}$ ice. Journal of Geophysical Research 103, 25809-25822.

Hampton, D. L., et al., 2005. An Overview of the Instrument Suite for the Deep Impact Mission. Space Science Reviews 117, 43-93.

Hanner, M. S., 1981. On the detectability of icy grains in the comae of comets. Icarus 47, 342-350.

Hapke, B., 1993. Theory of reflectance and emittance spectroscopy.

Jewitt, D. C., Luu, J., 2004. Crystalline water ice on the Kuiper belt object (50000) Quaoar. Nature 432, 731-733.

Kawakita, H., et al., 2004. Evidence of Icy Grains in Comet C/2002 T7 (LINEAR) at 3.52 AU. The Astrophysical Journal 601, L191-L194. 
Kelley, M. S., et al., 2013. A distribution of large particles in the coma of Comet 103P/Hartley 2. Icarus 222, 634-652.

Klaasen, K., et al., 2008. Deep Impact instrument calibration. Review of Scientific Instruments 79,77 .

Klaasen, K. P., et al., 2013. EPOXI instrument calibration. Icarus 225, 643-680.

Kurucz, R. L., 1995. The solar irradiance by computation. In: Anderson, G. P., Picard, R. H., Chetwind, J. H. (Eds.), Proceedings of the 17th Annual Conference on Atmospheric Transmission Models, PL-TR-95-2060. pp. 333-334.

Lindler, D. J., A'Hearn, M. F., Besse, S., Carcich, B., Hermalyn, B., Klaasen, K. P., 2013. Interpretation of results of deconvolved images from the Deep Impact spacecraft High Resolution Instrument. Icarus 222, 571-579.

Lisse, C. M., et al., 2009. Spitzer Space Telescope Observations of the Nucleus of Comet 103P/Hartley 2. Publications of the Astronomical Society of the Pacific $121,968-975$.

Mastrapa, R. M., Bernstein, M. P., Sandford, S. A., Roush, T. L., Cruikshank, D. P., Ore, C. M. D., 2008. Optical constants of amorphous and crystalline $\mathrm{H}_{2} \mathrm{O}$-ice in the near infrared from 1.1 to $2.6 \mu \mathrm{m}$. Icarus $197,307-320$.

Mastrapa, R. M., Sandford, S. A., Roush, T. L., Cruikshank, D. P., Dalle Ore, C. M., 2009. Optical Constants of Amorphous and Crystalline $\mathrm{H}_{2} \mathrm{O}$-ice: 2.5-22 $\mu \mathrm{m}\left(4000-455 \mathrm{~cm}^{-1}\right)$ Optical Constants of $\mathrm{H}_{2} \mathrm{O}$-ice. The Astrophysical Journal 701, 1347-1356. 
McLaughlin, S. A., Carcich, B., Sackett, S. E., Klaasen, K. P., 2013. EPOXI 103P/HARTLEY2 ENCOUNTER - HRII CALIBRATED SPECTRA V2.0, DIF-C-HRII-3/4-EPOXI-HARTLEY2-V2.0. NASA Planetary Data System.

Mumma, M. J., Charnley, S. B., 2011. The Chemical Composition of CometsEmerging Taxonomies and Natal Heritage. Annual Review of Astronomy and Astrophysics 49, 471-524.

Mumma, M. J., Weissman, P. R., Stern, S. A., 1993. Comets and the origin of the solar system - Reading the Rosetta Stone. In: Levy, E. H., Lunine, J. I. (Eds.), Protostars and Planets III. pp. 1177-1252.

Protopapa, S., et al., 2011. Size distribution of icy grains in the coma of 103P/Hartley 2. In: EPSC-DPS Joint Meeting 2011. p. 585.

Schleicher, D. G., Bair, A. N., 2011. The Composition of the Interior of Comet 73P/Schwassmann-Wachmann 3: Results from Narrowband Photometry of Multiple Components. The Astronomical Journal 141, 177.

Stern, S. A., 2003. The evolution of comets in the Oort cloud and Kuiper belt. Nature 424, 639-642.

Sunshine, J. M., et al., 2006. Exposed Water Ice Deposits on the Surface of Comet 9P/Tempel 1. Science 311, 1453-1455.

Sunshine, J. M., et al., 2011a. Icy Grains in Comet 103P/Hartley 2. In: Lunar and Planetary Institute Science Conference Abstracts. Vol. 42 of Lunar and Planetary Institute Science Conference Abstracts. p. 2292. 
Sunshine, J. M., et al., 2011b. Water Ice on Comet 103P/Hartley 2. In: EPSC-DPS Joint Meeting 2011. p. 1345.

Sunshine, J. M., et al., 2007. The distribution of water ice in the interior of Comet Tempel 1. Icarus 190, 284-294.

Tozzi, G. P., Lara, L. M., Kolokolova, L., Boehnhardt, H., Licandro, J., Schulz, R., 2004. Sublimating components in the coma of comet $\mathrm{C} / 2000 \mathrm{WM}_{1}$ (LINEAR). Astronomy and Astrophysics 424, 325-330.

Warren, S. G., Brandt, R. E., 2008. Optical constants of ice from the ultraviolet to the microwave: A revised compilation. Journal of Geophysical Research (Atmospheres) 113, 14220.

Weissman, P. R., 1986. Are cometary nuclei primordial rubble piles? Nature 320, 242-244.

Weissman, P. R., Asphaug, E., Lowry, S. C., 2004. Structure and density of cometary nuclei. pp. 337-357.

Weissman, P. R., Stern, S. A., 1997. Physical Processing of Cometary Nuclei. Tech. rep.

Whipple, F. L., 1951. A Comet Model. II. Physical Relations for Comets and Meteors. Astrophysical Journal 113, 464.

Yang, B., Jewitt, D., Bus, S. J., 2009. Comet 17P/Holmes in Outburst: The Near Infrared Spectrum. The Astronomical Journal 137, 4538-4546. 


\section{Appendix A. Scattered Flux}

The cometary flux in the wavelength range covered by the DI HRI-IR spectrometer is the sum of the scattered $\left(F_{\text {scat }}\right)$ and thermal $\left(F_{\text {thermal }}\right)$ components. We describe below how to estimate $F_{\text {scat }}$ and its contribution to the reflectance.

Consider a body at a distance $r$ from the Sun, of radius $R$. The total energy incident on the surface is

$$
L_{i n}=\pi R^{2} \frac{L_{\odot}}{4 \pi r^{2}}=\frac{L_{\odot} R^{2}}{4 r^{2}}
$$

where $L_{\odot}$ is the luminosity of the Sun. Only part of the incident flux is scattered back. The Bond Albedo A (or spherical albedo) is defined as the ratio of the emergent luminosity to the integrated incident flux $(0 \leq A \leq 1)$. Therefore, the luminosity is

$$
L_{\text {out }}=A L_{\text {in }}=\frac{A L_{\odot} R^{2}}{4 r^{2}}
$$

The target-observer distance is $\Delta$. If radiation is scattered isotropically, the observed flux is

$$
F_{\text {scat }}=\frac{L_{\text {out }}}{4 \pi \Delta^{2}} .
$$

If we assume that the reflecting object is a homogeneous sphere, the distribution of the reflected radiation only depends on the phase angle $\alpha$. Thus we can express the flux density observed at a distance $\Delta$ as

$$
F_{\text {scat }}=K \Phi(\alpha) \frac{L_{\text {out }}}{4 \pi \Delta^{2}}
$$

The function $\Phi$ is called phase function. It is normalized so that $\Phi(\alpha=0)=1$. $K$ is a normalization constant defined such that

$$
\int_{S} K \Phi(\alpha) \frac{L_{\text {out }}}{4 \pi \Delta^{2}} d S=L_{\text {out }}
$$


where the integration is extended over the surface of radius $\Delta$. Substituting Eq. A.2 in Eq A.4 we have

$$
F_{\text {scat }}=K \Phi(\alpha) \frac{A L_{\odot} R^{2}}{16 \pi \Delta^{2} r^{2}}
$$

The geometric albedo, $p$, is the ratio of the flux density of a body at phase angle $\alpha=0$ to the flux density of a perfect Lambert disk of the same radius and same distance as the body, but illuminated and observed perpendicularly. We have

$$
p=\frac{K A}{4}
$$

If we express $F_{\text {scat }}$ in terms of $p$, we obtain

$$
F_{\text {scat }}=\frac{p}{\pi} \Phi(\alpha) \frac{L_{\odot} R^{2}}{4 r^{2} \Delta^{2}} .
$$

The solar flux at a heliocentric distance $r_{1}=1 \mathrm{AU}$ is

$$
F_{\odot}=\frac{L_{\odot}}{4 \pi}
$$

We have then

$$
\frac{F_{\text {scat }}}{F_{\odot}}=\frac{\Phi(\alpha) p R^{2}}{r^{2} \Delta^{2}} .
$$

If we now consider the case of grains in a cometary coma, and we indicate with $C$ the total cross section of the cometary grains within the slit, the previous formula can be written as:

$$
\frac{F_{\text {scat }}}{F_{\odot}}=\frac{\Phi(\alpha) C p}{\pi r^{2} \Delta^{2}}
$$

The reflectance is defined as

$$
R=\frac{\pi I r^{2}}{F_{\odot}}
$$

where $I$ is the radiance (flux per unit solid angle). The contribution of the scattered flux to the reflectance, indicated as $R_{\text {scat }}$, is given by

$$
R_{\text {scat }}=\frac{\pi I_{\text {scat }} r^{2}}{F_{\odot}}=\frac{\pi F_{\text {scat }} r^{2}}{\Omega F_{\odot}}
$$


The solid angle of the coma portion we are observing is $\sigma / \Delta^{2}, \sigma$ being the geometric cross section. We have then

$$
R_{\text {scat }}=\frac{\pi F_{\text {scat }} \Delta^{2} r^{2}}{\sigma F_{\odot}}
$$

Considering A.11, and taking into account that the ratio between the scattering cross section and the geometric cross section is the filling factor $f$, we have

$$
R_{\text {scat }}=f \Phi(\alpha) p
$$


Table 1: Characteristics of the HRI-IR scans analyzed in this paper.

\begin{tabular}{ccc}
\hline Exposure ID & 5006000 & 5007002 \\
\hline Time at mid-scan at spacecraft & $2010-11-04$ 14:07:08 & $23010-11-04$ 14:23:10 \\
Time after CA [min] & 7 & 30 \\
Number of Commanded Frames & 56 & ALTFF \\
Mode name & ALTFF $^{a}$ & 1441 \\
Total integration time per frame [msec] & 1441 & 173 \\
Spatial resolution at mid-scan [m/pixel] & 55 & 93 \\
Phase angle [deg] & 92 & 17295 \\
Spacecraft-comet distance [km] & 5478 &
\end{tabular}

Table 2: Characteristics of the MRI-VIS and HRI-VIS data used in this paper as context images for the IR data.

\begin{tabular}{ccccc}
\hline Exposure ID & 5006046 & 5007065 & 5006036 & 5007064 \\
\hline Instrument & MRI-VIS & MRI-VIS & HRI-VIS & HRI-VIS \\
Time (2010-11-04) & $14: 06: 31$ & $14: 25: 11$ & $14: 06: 57$ & $14: 24: 58$ \\
Time after CA [min] & 7 & 25 & 7 & 25 \\
Total integration time per frame [msec] & 500 & 600 & 150 & 175 \\
Spatial resolution [m/pixel] & 50 & 188 & 11 & 37 \\
Filter & CLEAR1 & CLEAR1 & CLEAR1 & CLEAR1 \\
\hline
\end{tabular}


Table 3: Modeling Solutions

\begin{tabular}{|c|c|c|c|c|c|c|c|c|c|c|c|}
\hline Model & $P_{\mathrm{H}_{2} \mathrm{O}^{b}}$ & $\begin{array}{c}T_{\mathrm{H}_{2} \mathrm{O}} \\
(\mathrm{K})\end{array}$ & \multicolumn{2}{|c|}{$\begin{array}{c}\mathrm{H}_{2} \mathrm{O} \text {-ice } \\
\text { Optical Constants }\end{array}$} & $\begin{array}{c}<D_{H_{2} \mathrm{O}}> \\
(\mu \mathrm{m})\end{array}$ & $\begin{array}{c}<D_{A C}> \\
(\mu \mathrm{m})\end{array}$ & $\begin{array}{c}F_{\mathrm{H}_{2} \mathrm{O}} \\
(\%)\end{array}$ & $\begin{array}{l}T_{c} \\
(\mathrm{~K})\end{array}$ & $f$ & $f_{e}$ & $\chi_{\text {red }}^{2}$ \\
\hline BOX A Model1 & $\mathrm{I}_{c}$ & 266 & Warren and Brandt & 2008 & $1^{a}$ & $1^{a}$ & $<0.1$ & $315 \pm 3$ & $0.0063 \pm 0.0001$ & $0.0032 \pm 0.0006$ & $10.4 \pm 0.4$ \\
\hline BOX A Model2 & $\mathrm{I}_{c}$ & 266 & Warren and Brandt & 2008 & $1^{a}$ & $2^{a}$ & $<0.1$ & $318 \pm 3$ & $0.0077 \pm 0.0002$ & $0.0029 \pm 0.0006$ & $11.4 \pm 0.3$ \\
\hline BOX A Model3 & $\mathrm{I}_{c}$ & 266 & Warren and Brandt & 2008 & $1^{a}$ & $5^{a}$ & $<0.1$ & $322 \pm 3$ & $0.0082 \pm 0.0002$ & $0.0026 \pm 0.0005$ & $12.4 \pm 0.3$ \\
\hline BOX B Model1 & $\mathrm{I}_{c}$ & 266 & Warren and Brandt & 2008 & $1^{a}$ & $1^{a}$ & $5.1 \pm 0.2$ & $306 \pm 4$ & $0.0166 \pm 0.0003$ & $0.0058 \pm 0.0009$ & $16 \pm 1$ \\
\hline BOX B Model2 & $\mathrm{I}_{c}$ & 266 & Warren and Brandt & 2008 & $0.82 \pm 0.07$ & $1^{a}$ & $5.3 \pm 0.2$ & $307 \pm 4$ & $0.0163 \pm 0.0004$ & $0.0056 \pm 0.0009$ & $16 \pm 1$ \\
\hline BOX B Model3 & $\mathrm{I}_{c}$ & 266 & Warren and Brandt & 2008 & $2^{a}$ & $1^{a}$ & $5.0 \pm 0.2$ & $308 \pm 4$ & $0.0173 \pm 0.0004$ & $0.0054 \pm 0.0009$ & $17 \pm 2$ \\
\hline BOX B Model4 & $\mathrm{I}_{c}$ & 266 & Warren and Brandt & 2008 & $5^{a}$ & $1^{a}$ & $4.9 \pm 0.2$ & $311 \pm 4$ & $0.0184 \pm 0.0004$ & $0.0049 \pm 0.0008$ & $22 \pm 2$ \\
\hline BOX в Model5 & $\mathrm{I}_{c}$ & 266 & Warren and Brandt & 2008 & $10^{a}$ & $1^{a}$ & $4.7 \pm 0.1$ & $313 \pm 3$ & $0.0196 \pm 0.0004$ & $0.0043 \pm 0.0004$ & $30 \pm 3$ \\
\hline BOX B Model6 & $\mathrm{I}_{c}$ & 266 & Warren and Brandt & 2008 & $100^{a}$ & $1^{a}$ & $3.7 \pm 0.1$ & $301 \pm 3$ & $0.0255 \pm 0.0006$ & $0.0063 \pm 0.0006$ & $73 \pm 5$ \\
\hline BOX B Model7 & $\mathrm{I}_{a}$ & 120 & Mastrapa et al. 2 & 2009 & $1^{a}$ & $1^{a}$ & $5.0 \pm 0.2$ & $307 \pm 4$ & $0.0167 \pm 0.0003$ & $0.0056 \pm 0.0009$ & $16 \pm 1$ \\
\hline BOX B Model8 & $\mathrm{I}_{c}$ & 120 & Mastrapa et al. 2 & 2009 & $1^{a}$ & $1^{a}$ & $5.2 \pm 0.2$ & $312 \pm 3$ & $0.0165 \pm 0.0004$ & $0.0044 \pm 0.0004$ & $17 \pm 1$ \\
\hline
\end{tabular}

${ }^{a}$ The particle grain sizes are fixed in the modeling

${ }^{b}$ The water ice phase.

Table 4: Values obtained from the fit of the ice and dust column density vs. $\rho$.

\begin{tabular}{cccccccc}
\hline Component & function & jet & $\begin{array}{c}\log _{10} \alpha \\
(\mathrm{m})\end{array}$ & $k$ & $\rho^{\prime}$ & $\log _{10} \beta$ & $\begin{array}{c}L \\
(\mathrm{~m})\end{array}$ \\
\hline Dust & $\alpha \rho^{k}$ & $\mathrm{~J}_{1}$ & $13.35 \pm 0.08$ & $-0.99 \pm 0.02$ & & & \\
Dust & $\alpha \rho^{k}$ & $\mathrm{~J}_{4}$ & $13.07 \pm 0.04$ & $-1.00 \pm 0.01$ & & & \\
Ice & $\frac{\beta}{\rho} e^{-\frac{\rho-\rho^{\prime}}{L}}$ & $\mathrm{~J}_{1}$ & & & 935 & $12.38 \pm 0.02$ & $636 \pm 17$ \\
Ice & $\frac{\beta}{\rho} e^{-\frac{\rho-\rho^{\prime}}{L}}$ & $\mathrm{~J}_{4}$ & & & 165 & $12.12 \pm 0.05$ & $231 \pm 8$ \\
\hline
\end{tabular}


$\mathrm{CA}+7 \mathrm{~min}$

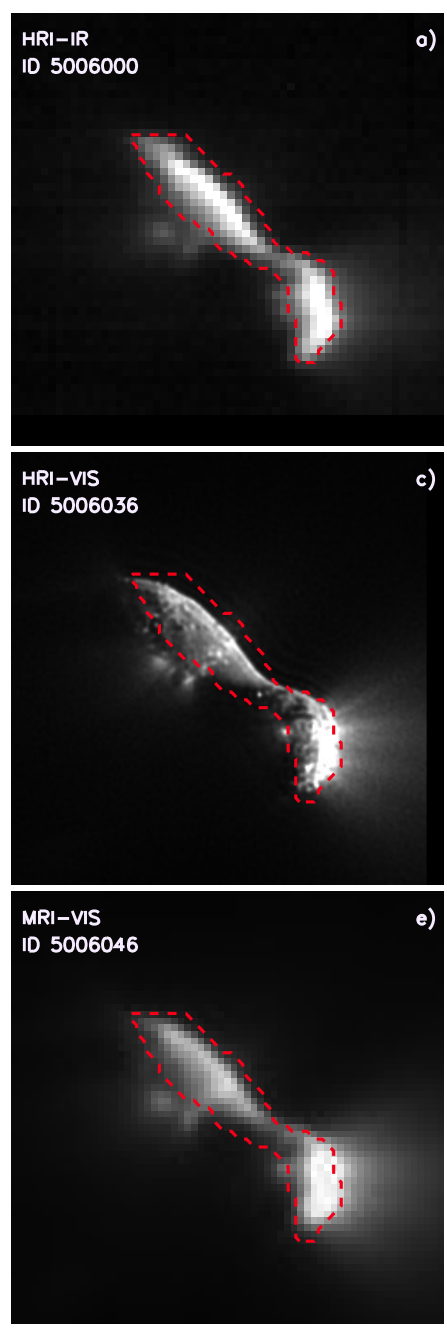

$\mathrm{CA}+23 \mathrm{~min}$

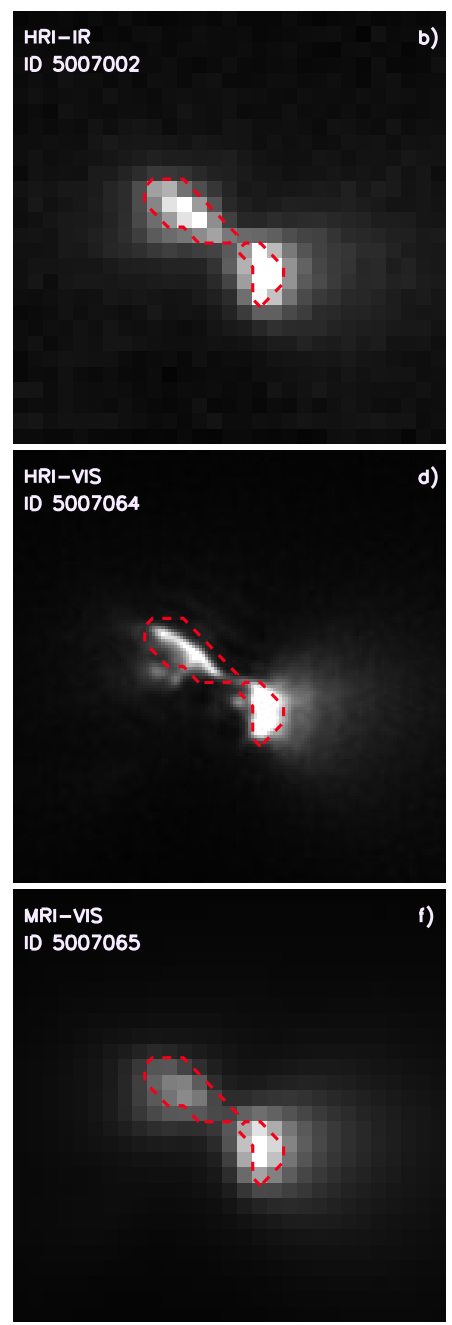

Figure 1: The HRI-IR spectral cubes acquired 7 and 23 min post-CA (see Table 11) at $2.5 \mu \mathrm{m}$ are displayed in panels (a) and (b), respectively. Panels (c) and (d) show the context images of Hartley 2 taken with the high resolution camera HRIVIS, corresponding to the ID sequences 5006036 and 5007064, respectively. The images have been deconvolved [Lindler et al., 2013]. The bottom 2 panels display the MRI-VIS context images. Table 2lists the main characteristics of the HRI-VIS and MRI-VIS context images shown in this Figure. The dashed red line contours the illuminated portion of the nucleus that is masked for our analysis (for details see text). The Sun is to the right. See the electronic version of the Journal for a color version of this figure. 

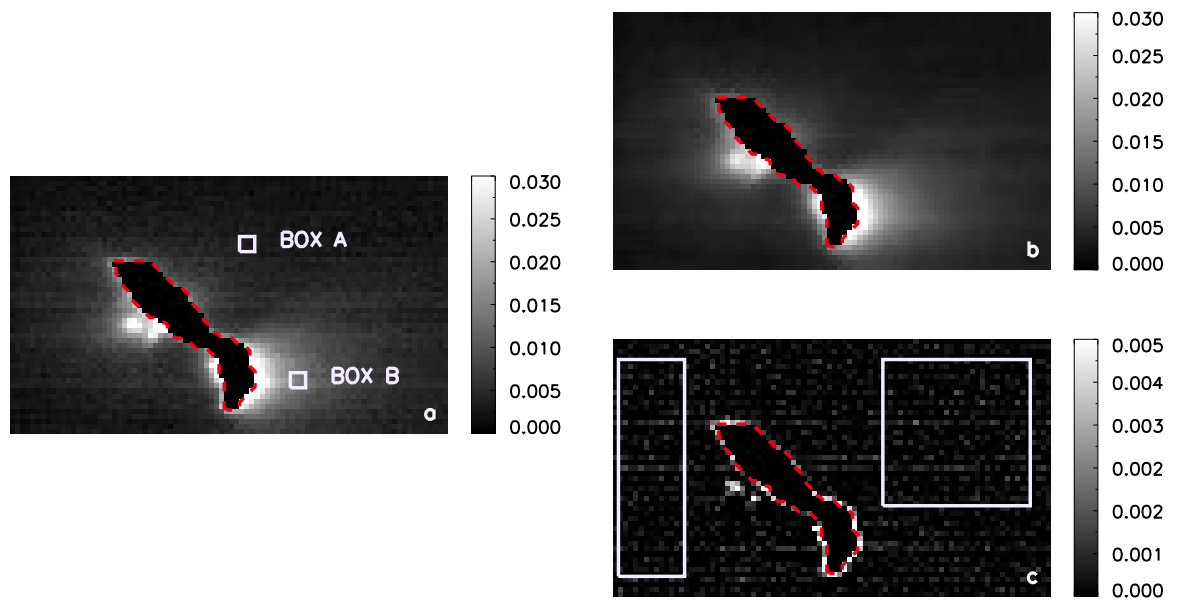

Figure 2: Radiance error determination. (a) Radiance map acquired 7 min postCA (ID 5006000) at $2.5 \mu \mathrm{m}$. The color bar indicates the line of sight radiance values ( $\mathrm{W} \mathrm{m}^{-2} \mathrm{sr}^{-1} \mu \mathrm{m}^{-1}$ ). The white boxes represent the regions sampled to produce the reflectance spectra in Figure 3. (b) Radiance map after mean filtering with a $3 \times 3$ pixel square kernel. (c) Radiance background map obtained by subtracting (b) from (a). The white boxes surround the regions used to compute the mean error (see text for details). In all maps, the nucleus has been masked and the dashed red line contours the nucleus. The Sun is to the right. See the electronic version of the Journal for a color version of this figure. 


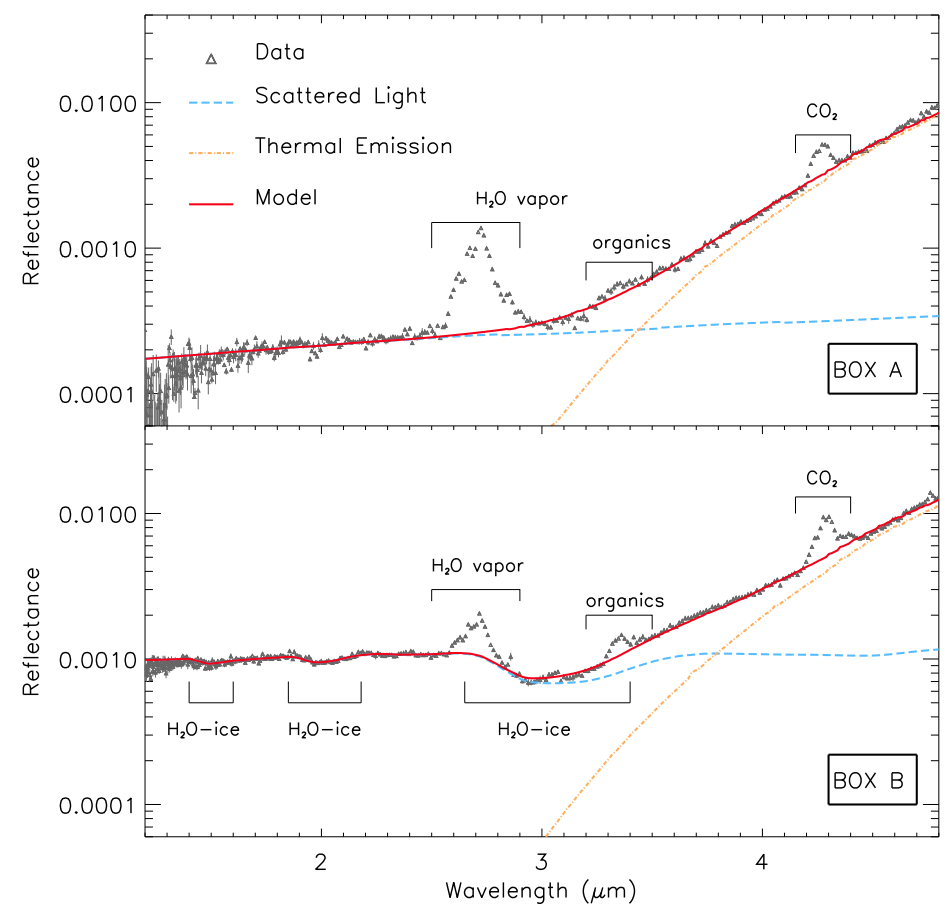

Figure 3: Comet 103P/Hartley 2 reflectance spectra (gray triangles) over the wavelength range $1.2-4.8 \mu \mathrm{m}$. Top and bottom panels show the spectrum (triangles) obtained performing the resistant mean (threshold 2.5 $\sigma$ ) of the 9 reflectance spectra in box A (top panel) and B (bottom panel) of Figure 2 (panel a), respectively. The modeling (solid red line) is the sum of the scattered solar radiation (dashed blue line) and a blackbody function (dash-dot orange line). The spectrum shows clearly the water vapor, organic, and carbon dioxide emissions at $2.7 \mu \mathrm{m}, 3.3 \mu \mathrm{m}$, and $4.3 \mu \mathrm{m}$, respectively, which are not fit by the model as expected. The species responsible for the emission and absorption features are marked in the plots. See the electronic version of the Journal for a color version of this figure. 


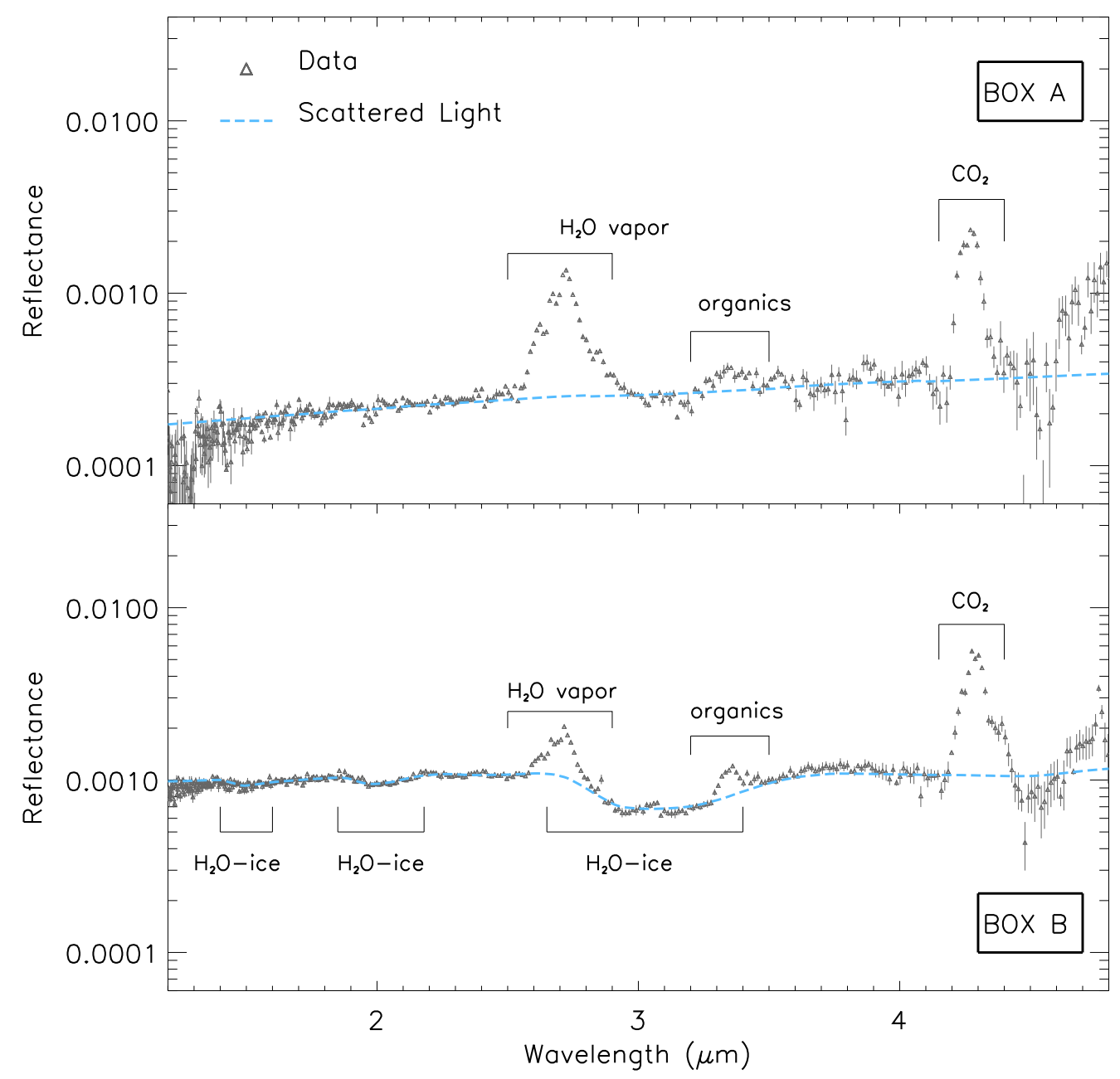

Figure 4: Comet 103P/Hartley 2 reflectance spectra shown in Figure 3 after the removal of the thermal models (gray triangles). Overplotted is the scattered model (dashed blue line). See the electronic version of the Journal for a color version of this figure. 


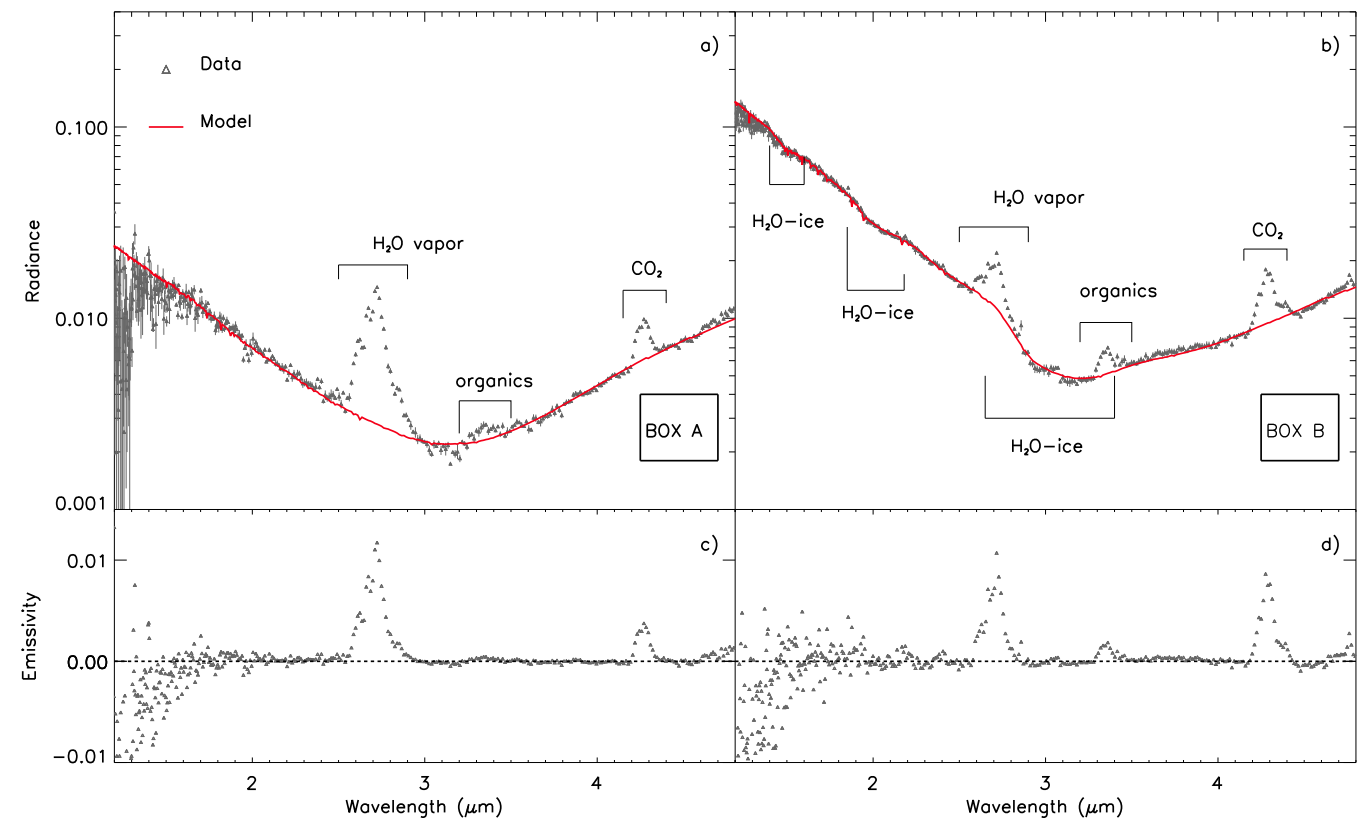

Figure 5: Panels (a) and (b) show the radiance spectrum (triangles) of comet 103P/Hartley 2 over the wavelength range $1.2-4.8 \mu \mathrm{m}$, extracted in boxes $\mathrm{A}$ and $\mathrm{B}$ in panel (a) of Figure 2, respectively. Overplotted is the model (solid red line) shown in Figure 3 converted into radiance. Both spectra clearly display the water vapor, organic, and carbon dioxide emissions, which are not fit by the model, as expected. Panels (c) and (d) display the emissivity spectrum or equivalently the residuals between the model and the data shown in panels (a) and (b), respectively. See the electronic version of the Journal for a color version of this figure. 


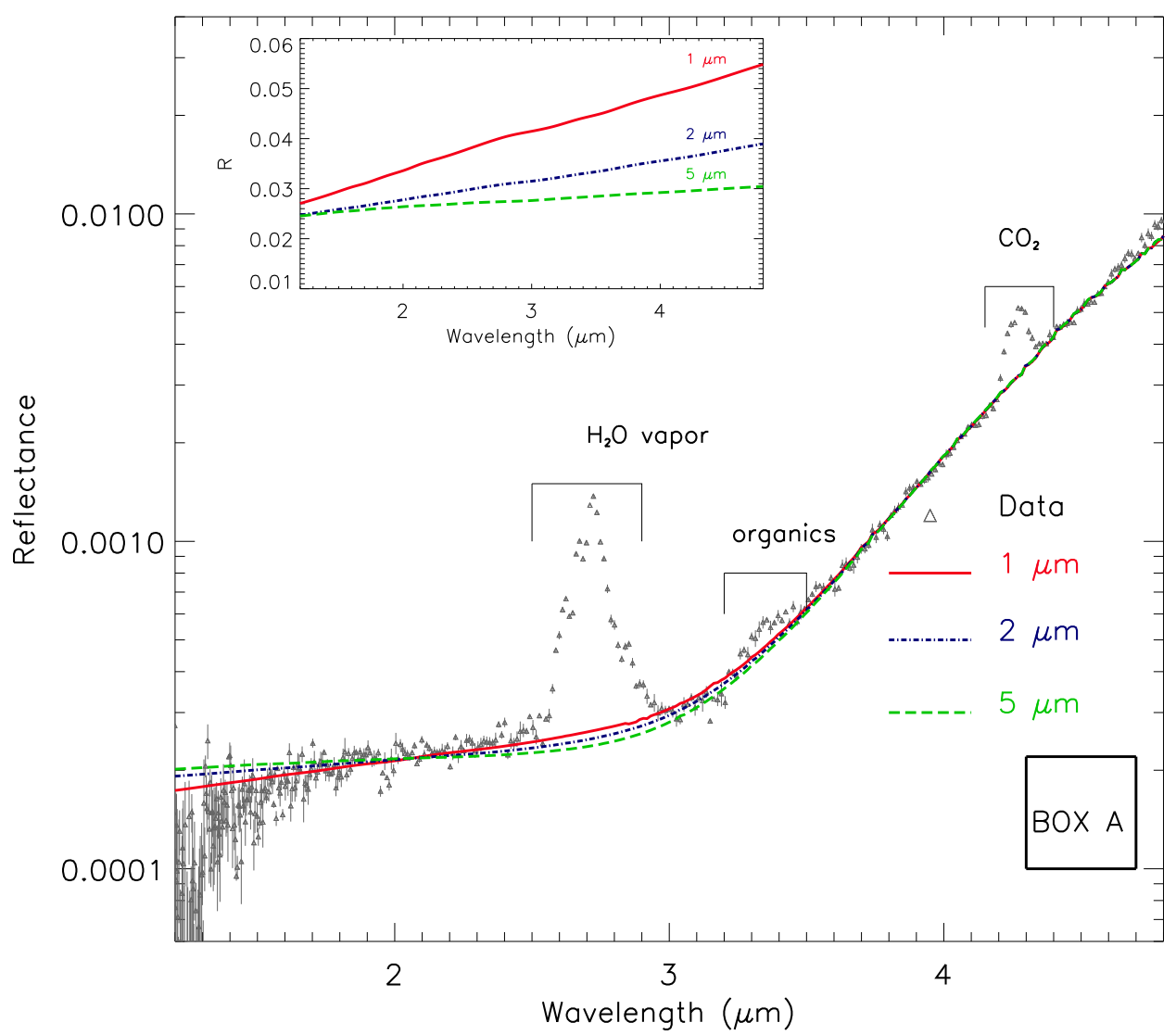

Figure 6: The reflectance spectrum of comet 103P/Hartley 2 from the top panel of Figure 3 compared with synthetic spectra obtained assuming amorphous carbon on the order of 1 (solid red line), 2 (dash-dot purple line), and 5 (dashed green line) $\mu \mathrm{m}$ in diameter. The modeling details are reported in Table 3. The inset panel shows synthetic reflectance spectra of various amorphous-carbon grains in the wavelength range covered by the HRI-IR instrument. See the electronic version of the Journal for a color version of this figure. 


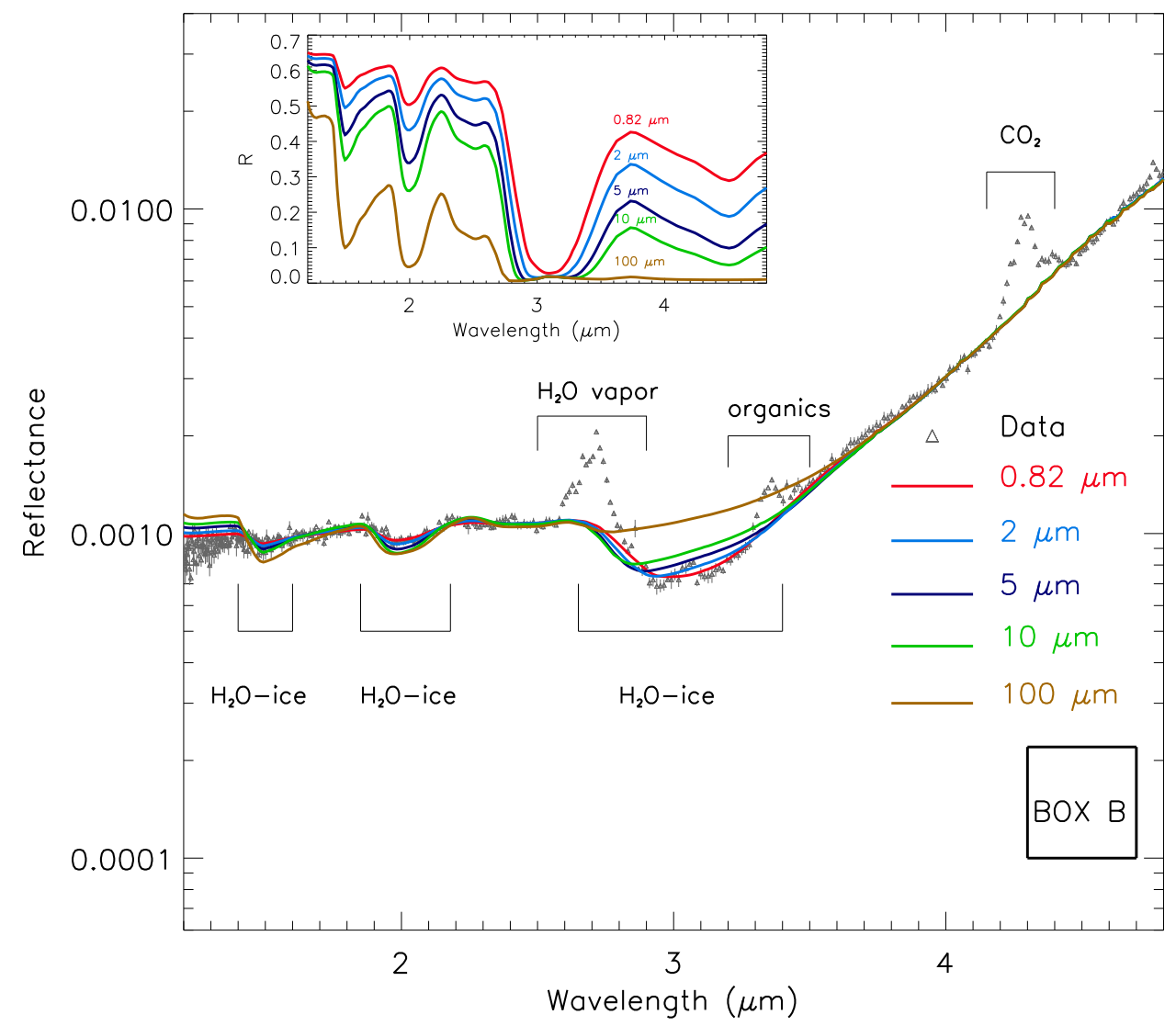

Figure 7: The reflectance spectrum of comet 103P/Hartley 2 from the bottom panel of Figure 3 compared with synthetic spectra obtained assuming an areal mixture of amorphous-carbon and water ice grains on the order of 0.82 (red), 2 (blue), 5 (purple), 10 (green), and 100 (brown) $\mu \mathrm{m}$. The modeling details are reported in Table 3. The inset panel shows synthetic reflectance spectra of various sized water ice grains in the wavelength range covered by the HRI-IR instrument. 


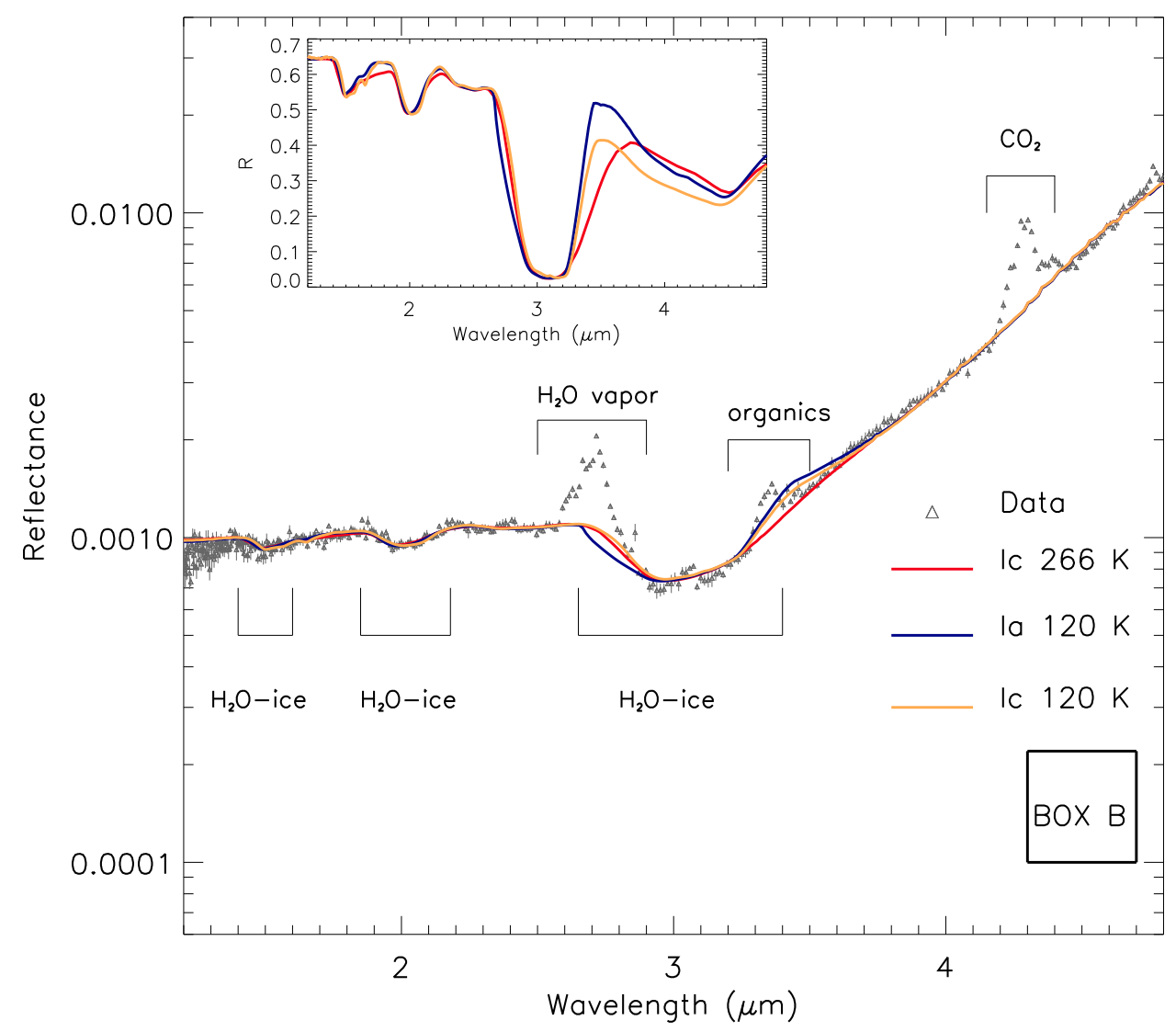

Figure 8: The reflectance spectrum of comet 103P/Hartley 2 from the bottom panel of Figure 3 compared with synthetic spectra obtained assuming an areal mixture of amorphous carbon and water ice at different phase and temperatures. We compare models obtained with optical constants from Mastrapa et al. [2009] of amorphous (Ia) and crystalline (Ic) water ice at $120 \mathrm{~K}$ (purple and yellow line, respectively), and with optical constants of Warren and Brandt [2008] for crystalline ice at $266 \mathrm{~K}$ (red line). The inset panel shows synthetic reflectance spectra of $100 \%$ water ice $(1 \mu \mathrm{m}$ particle size $)$ computed using the same set of optical constants. 


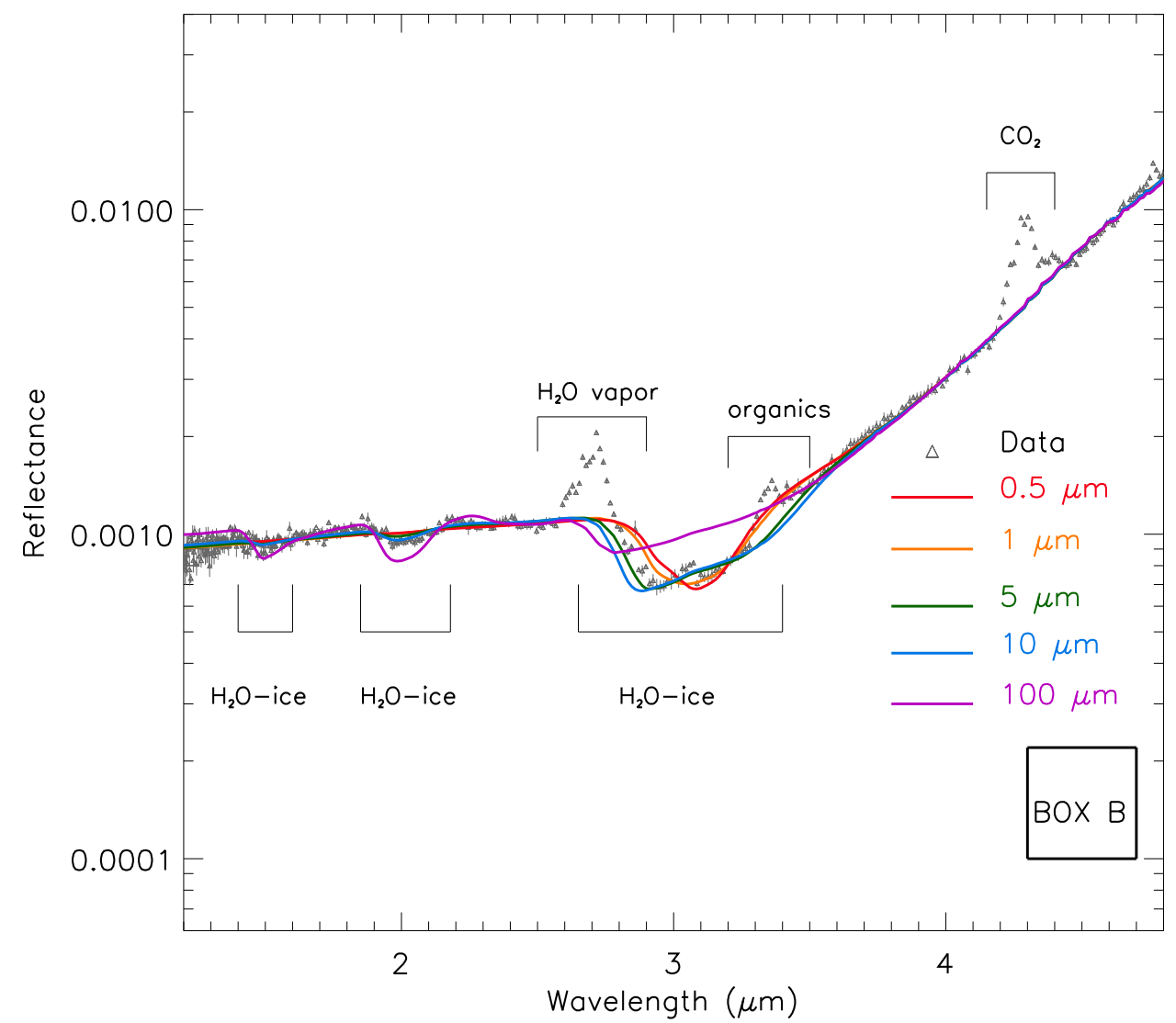

Figure 9: Models of the water ice-rich spectrum of Hartley 2 observed 7 min postCA assuming an intimate mixture. All sizes of water ice lead to poor solutions when intimately mixed with dark refractory materials. 

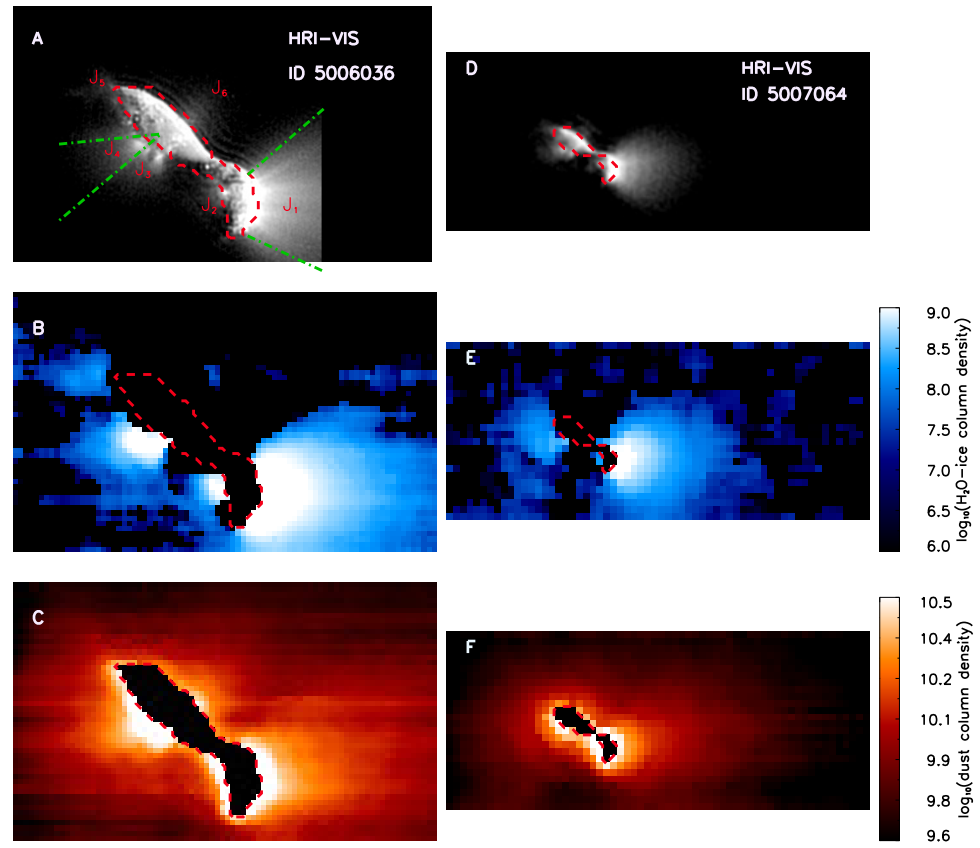

Figure 10: Two sets of observations of Hartley 2 separated by 16 min. Water ice and refractories are detected in the innermost coma of Hartley 2. The Sun is to the right. The dashed red line contours the masked comet nucleus. (A to C) Data acquired $7 \mathrm{~min}$ post-CA. (A) HRI-VIS context image at $\sim 11 \mathrm{~m} /$ pixel. Overlaid in dash-dot green are the jet areas sampled to produce the profiles in Figure 12. (B) Water ice column density expressed in number of particles per square meter derived from the 5006000 HRI-IR scan at $55 \mathrm{~m} /$ pixel. The lower limit for water ice detection is $6 \times 10^{6}$ particles $/ \mathrm{m}^{2}$. The regions in black contain little to no water ice (see text for details). (C) Dust column density expressed in number of particles per square meter. (D to F) Data acquired 23 min post-CA; images matching (A) to (C) at a spatial resolution of $37 \mathrm{~m} / \mathrm{pixel}$ in the VIS (ID 5007064) and $173 \mathrm{~m} / \mathrm{pixel}$ in the IR (scan 5007002). See the electronic version of the Journal for a color version of this figure. 

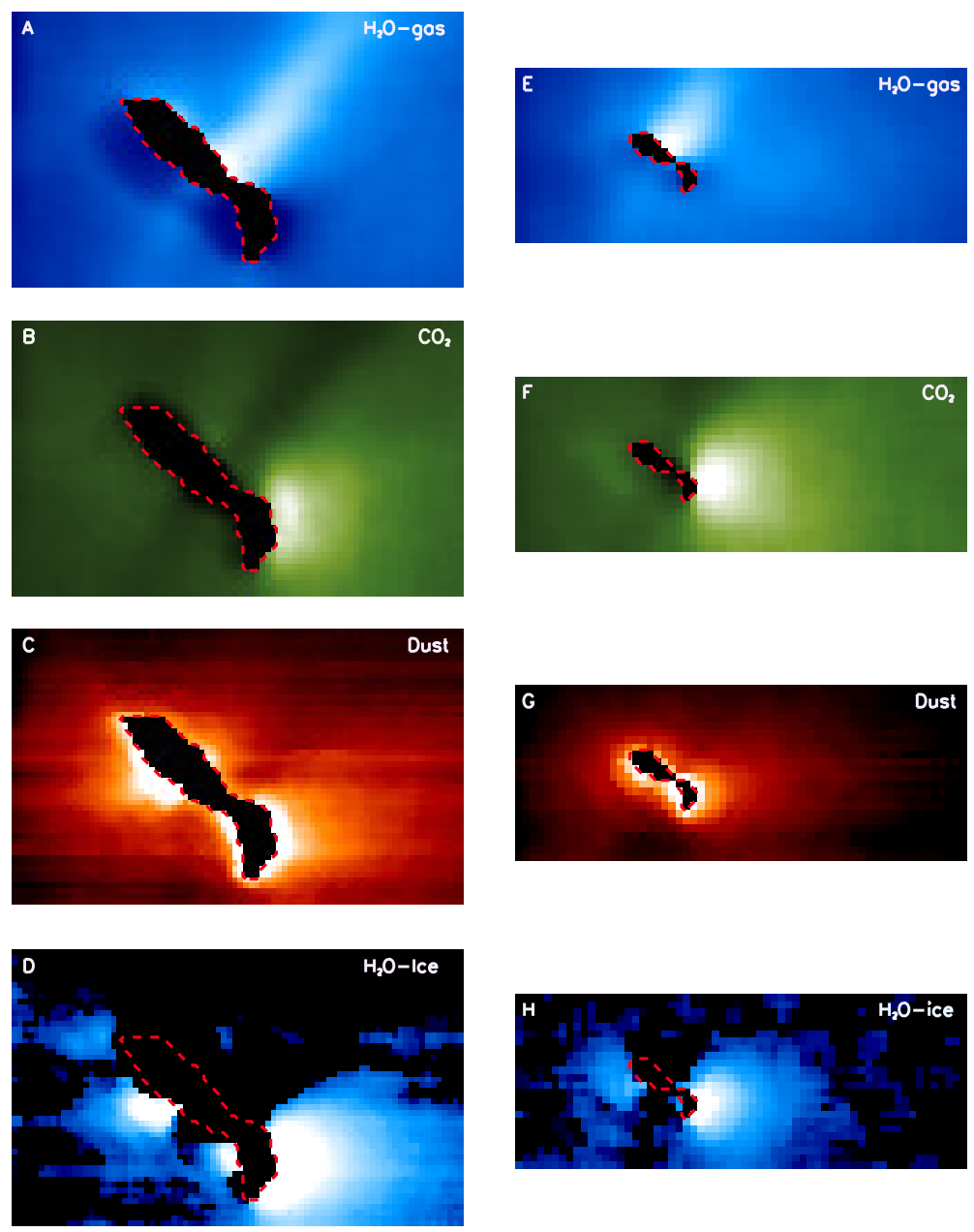

Figure 11: Distribution of $\mathrm{H}_{2} \mathrm{O}$-gas, $\mathrm{CO}_{2}$, Dust, and $\mathrm{H}_{2} \mathrm{O}$-ice in the innermost coma of Hartley 2 as observed 7 min (left column) and 23 min (right column) post-CA. The Sun is to the right. The dashed red line contours the masked comet nucleus. Enhancements in the $\mathrm{CO}_{2}$ and $\mathrm{H}_{2} \mathrm{O}$-gas relative abundances are not correlated. On the other hand, a strong correlation between $\mathrm{CO}_{2}, \mathrm{H}_{2} \mathrm{O}$-ice, and dust is observed in the inner coma. In all panels white is relatively more abundant. See the electronic version of the Journal for a color version of this figure. 

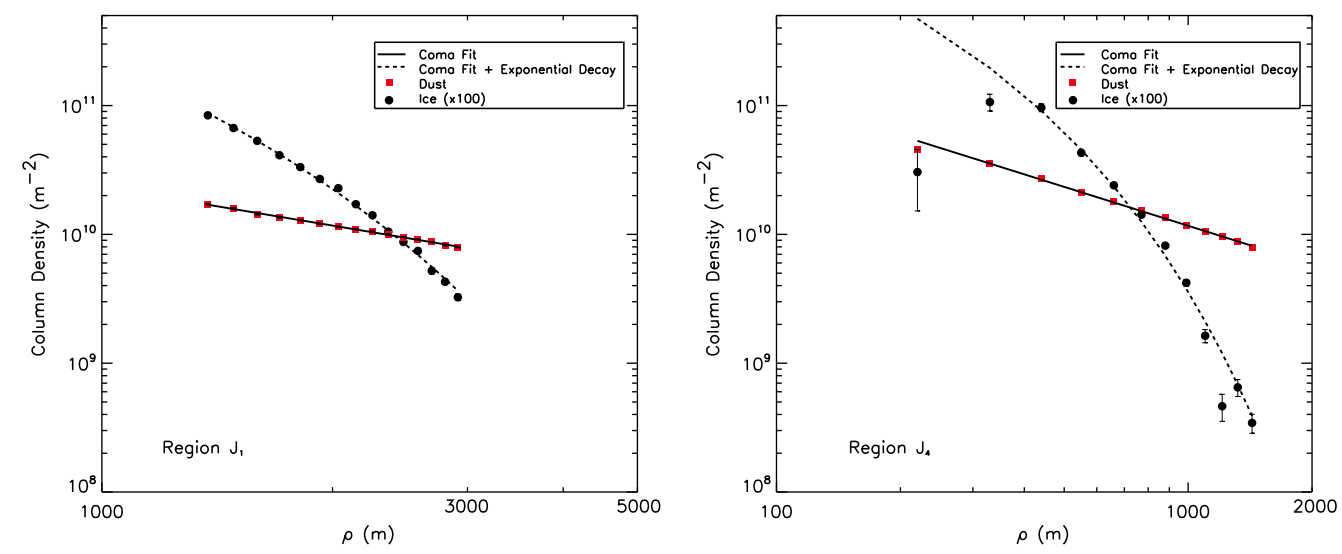

Figure 12: Column density profiles of the dust (squares) and water ice (circles) in the jets $\mathbf{J}_{1}$ (left panel) and $\mathbf{J}_{4}$ (right panel) delimited by the dash-dot green lines shown in panel (A) of Figure 10. Solid and dashed lines represent the models of the dust and water ice profiles, respectively. While the dust presents a constant outflow profile, a steeper profile is displayed by the water ice, which can be fit by an exponential decay (see text for details). See the electronic version of the Journal for a color version of this figure. 